\title{
Quantum quenches in a holographic Kondo model
}

\author{
Johanna Erdmenger, ${ }^{a, b}$ Mario Flory, ${ }^{a, c}$ Max-Niklas Newrzella, ${ }^{a}$ Migael Strydom ${ }^{a}$ and \\ Jackson M.S. Wu ${ }^{d}$ \\ ${ }^{a}$ Max-Planck-Institut für Physik (Werner-Heisenberg-Institut), \\ Föhringer Ring 6, 80805, Munich, Germany \\ ${ }^{b}$ Institut für Theoretische Physik und Astrophysik, Julius-Maximilians-Universität Würzburg, \\ Am Hubland, 97074 Würzburg, Germany \\ ${ }^{c}$ Institute of Physics, Jagiellonian University, \\ Eojasiewicza 11, 30-348 Kraków, Poland \\ ${ }^{d}$ Department of Physics and Astronomy, University of Alabama, \\ Tuscaloosa, AL 35487, U.S.A. \\ E-mail: jke@mpp.mpg.de, mflory@th.if.uj.edu.pl, maxnew@mpp.mpg.de, \\ migael.strydom@gmail.com, jknwgm13@gmail.com
}

ABSTRACT: We study non-equilibrium dynamics and quantum quenches in a recent gauge/ gravity duality model for a strongly coupled system interacting with a magnetic impurity with $\mathrm{SU}(N)$ spin. At large $N$, it is convenient to write the impurity spin as a bilinear in Abrikosov fermions. The model describes an RG flow triggered by the marginally relevant Kondo operator. There is a phase transition at a critical temperature, below which an operator condenses which involves both an electron and an Abrikosov fermion field. This corresponds to a holographic superconductor in $\mathrm{AdS}_{2}$ and models the impurity screening. We quench the Kondo coupling either by a Gaussian pulse or by a hyperbolic tangent, the latter taking the system from the condensed to the uncondensed phase or vice-versa. We study the time dependence of the condensate induced by this quench. The timescale for equilibration is generically given by the leading quasinormal mode of the dual gravity model. This mode also governs the formation of the screening cloud, which is obtained as the decrease of impurity degrees of freedom with time. In the condensed phase, the leading quasinormal mode is imaginary and the relaxation of the condensate is over-damped. For quenches whose final state is close to the critical point of the large $N$ phase transition, we study the critical slowing down and obtain the combination of critical exponents $z \nu=1$. When the final state is exactly at the phase transition, we find that the exponential ringing of the quasinormal modes is replaced by a power-law behaviour of the form $\sim t^{-a} \sin (b \log t)$. This indicates the emergence of a discrete scale invariance.

KEYWORDS: Gauge-gravity correspondence, Holography and condensed matter physics (AdS/CMT)

ARXIV EPRINT: 1612.06860 


\section{Contents}

1 Introduction 1

2 Setup 4

2.1 Action and equations of motion 4

2.2 Boundary behaviour and conditions on the Kondo coupling 6

3 Results $\quad 8$

3.1 Generic time evolution for phase transitions 8

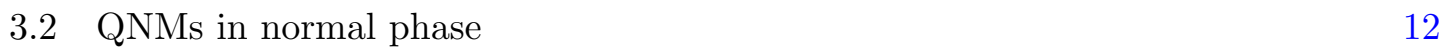

\begin{tabular}{ll}
3.3 & QNMs in condensed phase \\
\hline
\end{tabular}

$\begin{array}{ll}3.4 & \text { Evolution of the screening of the impurity } \\ \end{array}$

4 Critical behaviour $\quad 16$

$\begin{array}{lll}\text { 4.1 Critical slowing down } & 16\end{array}$

4.2 Power-law behaviour and discrete scale invariance at the critical point 18

5 Summary and outlook $\quad 20$

$\begin{array}{lll}5.1 \text { Summary } 20 & 20\end{array}$

$\begin{array}{ll}5.2 \text { Outlook } & 22\end{array}$

A Gauge fixing, coordinates and field redefinition 23

$\begin{array}{ll}\text { B Numerical evolution scheme } & 25\end{array}$

\section{Introduction}

The AdS/CFT correspondence [1-3] and its generalisations to a more general gauge/gravity duality provide a new approach for studying strongly correlated systems. One of the many applications of gauge/gravity duality is the study of non-equilibrium strongly coupled systems. This applies in particular to quantum quenches and to the subsequent relaxation to a new ground state. While this idea was first studied in relation to heavy-ion physics and the quark-gluon plasma $[4,5]$, more recently quenches have been considered for systems relevant to condensed matter as well $[6,7]$.

In this paper we use gauge/gravity duality to study quantum quenches for strongly coupled systems interacting with a magnetic impurity. In particular, we consider quenches in a recent holographic model [8] that describes the RG flow triggered by an impurity operator. This is a holographic variant of the well-known Kondo model with certain distinct features owed to considering a model for which a gravity dual may be constructed. As is standard in the AdS/CFT correspondence, the large $N$ limit needs to be taken to obtain a classical gravity dual. Thus in this model, the spin group of the impurity spin is $\mathrm{SU}(N)$. 
For constructing the gravity dual model of [8], it was important to note that large $N$ Kondo models are well-known in the condensed matter literature [9-11]. According to this approach, the spin is taken to be in a totally antisymmetric representation given by a Young tableau with $q$ boxes. In the large $N$ limit, it is convenient to introduce Abrikosov pseudo-fermions $\chi$ and to write the impurity field as a bilinear in these fermions, i.e. $S^{a}=\bar{\chi} T^{a} \chi$. Using Fierz identities and dropping terms subleading in $1 / N$, the Kondo interaction $\kappa J^{a} S^{a}$ with $J^{a}$ the electron current becomes $\kappa \mathcal{O O}^{\dagger}$ with $\mathcal{O}=\psi^{\dagger} \chi$. Here, $\kappa$ is the Kondo coupling, $\psi$ is an electron and $\chi$ an Abrikosov fermion as introduced above. This operator triggers a flow to an IR fixed point. The key point of the condensed-matter large $N$ Kondo model is thart the IR is characterised by a non-trivial condensate $\langle\mathcal{O}\rangle$, i.e. there is a critical temperature $T_{c}$, slightly smaller than the Kondo temperature $T_{K}$, below which this condensate forms $[10,11]$. The condensation corresponds to screening of the impurity by the Kondo cloud.

In the dual gravity model, according to [8] a related impurity system is described by a holographic superconductor in the following setting: the gravity dual action involves a Chern-Simons field in $2+1$ dimensions dual to the electron current. For simplicity, the number of channels (flavours) is taken to be $k=1$, which renders this Chern-Simons field Abelian. In addition, there is a propagating Abelian gauge field restricted to a $(1+1)$ dimensional subspace spanned by time and the holographic radial coordinate. The time component is dual to the charge density $\chi^{\dagger} \chi$. Moreover, there is complex scalar in the same $(1+1)$-dimensional subspace, which is dual to the operator $\mathcal{O}$. In the probe limit, all of these fields are embedded in a BTZ black hole spacetime, i.e. a black hole in $A d S_{3}$. The horizon of the black hole sets the temperature.

A central difference between the holographic model and the standard large $N$ Kondo model is that the electron gas is strongly coupled even before the interaction with the magnetic impurity is switched on. For describing the required RG flow, the strongly coupled theory is perturbed by the marginally relevant operator $\kappa \mathcal{O O}^{\dagger}$ with $\mathcal{O}=\psi^{\dagger} \chi$. Holographically, this perturbation is achieved by considering the gravity dual of a 'double trace' deformation as introduced in [12]. $\kappa$ diverges at a temperature scale $T_{K}$ which defines the Kondo temperature. Solving the equations of motion, a second-order (mean field) phase transition is found at a temperature $T_{c}$ just below $T_{K}$. Below $T_{c}$, the scalar acquires a non-trivial condensate. This is the gravity dual analogue of the phase transition in the condensed-matter large $N$ Kondo model: precisely the same operator $\psi^{\dagger} \chi$ condenses in both cases. Moreover, at low temperatures the charge density dual to the two-dimensional gauge field decreases, such that the dimension of the spin representation is decreased. This corresponds to the screening of the impurity. Also, the electrons are subject to a phase shift which is obtained from the Wilson loop involving the Chern-Simons field in $A d S_{3}$. The resistivity is obtained from an analysis of the leading irrelevant operator. Due to the large $N$ limit, the characteristic logarithmic behaviour at low temperatures is absent. Rather, the resistivity has a polynomial dependence on temperature with real exponent [8].

In [13-15], the model of [8] was extended to include the backreaction of the defect on the background geometry, which allows to the calculation of the entanglement entropy in particular. The two-impurity version of the model of [8] was studied in [16]. Recently, 
two-point functions for this model were calculated in [17, 18], where it was found that the spectral function displays a Fano resonance characteristic of scattering between a continuum and a localised resonance. In the screened phase where $\langle\mathcal{O}\rangle \neq 0$, the quasinormal modes (QNM) are purely imaginary and scale as $\omega \propto-i\langle\mathcal{O}\rangle^{2}$. This is characteristic of the Kondo resonance in the large $N$ limit.

In this paper, we restrict to the probe limit as in $[8,16]$. We consider time-dependent configurations in which the time-dependence of the Kondo coupling is chosen as an input. In particular, we consider both Gaussian pulses in the Kondo coupling $\kappa$ as well as tanhshaped transitions from the unscreened to the screened phase and vice-versa. We consider fast quenches, in which the quench time for the Kondo coupling is of order of the inverse Kondo temperature.

Our main result is that as generally expected in the holographic approach, the response of the system to quenches is dominated by the QNM. These fix the equilibration time for relaxation to the new ground state. In general, this relaxation time is independent of the original quench time. The QNMs ensure that the relaxation time is longer when the final state is closer to the phase transition. For relaxation to the critical state at the phase transition, as obtained when quenching the Kondo coupling to its critical value, the relaxation becomes polynomial rather than exponential. In this case, there is a critical slowing down and a damped log-periodic behaviour which may be a sign of discrete scale invariance.

For quenches to the screened phase we confirm that the leading QNM behaves as $\omega \propto-i\langle\mathcal{O}\rangle^{2}$ as seen in $[17,18]$. The fact that the leading QNM is imaginary implies that there are no oscillations about the new ground state, and the relaxation is overdamped. For larger values of the condensate, i.e. at very low temperatures, we see a different behaviour $\omega \propto-i \ln \langle\mathcal{O}\rangle$, which corresponds to a deviation from mean-field behaviour. The investigation of the zero-temperature behaviour will require a refinement of the model corresponding to stabilisation of the IR fixed point by a quartic contribution to the scalar potential on the gravity side, which we leave for the future.

In the model considered, the number of degrees of freedom at the impurity site is represented by the charge density of the Abrikosov fermions. This is related to the size of the spin representation. The charge density is holographically dual to the $A d S_{2}$ gauge field and may be written as the flux of the $A d S_{2}$ gauge field through the boundary of $A d S_{2}$. Evaluating this flux at the black hole horizon then gives a measure of the effective number of impurity degrees of freedom. In the condensed phase, we observe that this flux is reduced, which corresponds to a gravity dual realisation of the screening. In this paper, we study the time evolution of the flux after a Gaussian quench in the condensed phase and observe that it decays exponentially, which corresponds to an exponential decrease of the number of degrees of freedom after a quench. This models the time dependence of the Kondo cloud formation at the impurity site.

Quantum quenches in the standard SU(2) Kondo model were recently studied within condensed matter physics. These investigations include [19-23]. In particular, [19] deals with the study of a quantum quench caused by the absorption of a photon by a quantum dot, while [22] studies the universal behaviour of entanglement entropy after a quench of 
an impurity system. In [20], quenches in the pseudogap single-impurity Anderson model are investigated using numerical $R G$ techniques. The system reaches an equilibrium state at late times. Furthermore, the spatiotemporal formation of the Kondo cloud after a quench was simulated in [23], where an emergent lightcone structure is observed. The non-equilibrium correlation functions are determined by two different scales, initially by the lattice Fermi velocity and by the Kondo temperature at late time. In our model considered here, generically the Kondo temperature is the only scale. Generally, due to the large $N$ limit for the spin symmetry required in our holographic approach, comparison to the condensed matter results is possible only concerning a limit number of aspects, in particular due to the large $N$ phase transition which is not present in the $\mathrm{SU}(2)$ case. The large $N$ limit implies that in the screened (condensed) phase, there are no oscillations of the screening cloud and the relaxation of the condensate is over-damped. On the other hand, in $\mathrm{SU}(2)$ Kondo quenches such oscillations are frequently present, as seen for instance in [22].

The paper [7] considers quenches in 3+1-dimensional holographic superconductors with backreaction. Similarly to the present paper, for some parameter regimes an over-damped behaviour is found. In contrast to those results however, here we generically find overdamping whenever the final state is in condensed phase. This is due to the leading QNM being purely imaginary in this phase, as expected from the presence of a Kondo resonance. Moreover, [24] studies holographic quenches of a double-trace operator in arbitrary dimensions, and the corresponding critical exponents for quenches through the phase transition are obtained.

The structure of this paper is as follows: in section 2, we briefly describe the setup of the holographic Kondo model of [8]. Some additional details about the analytical and numerical treatment of the resulting equations of motion are relegated to appendices $\mathrm{A}$ and B. We then summarise our results in section 3. We furthermore study quenches in the normal phase in section 3.2, as well as in the condensed phase in section 3.3. In section 4.1 we study the phenomenon of critical slowing down near the phase transition. In section 4.2, this leads us to a study of the late-time behaviour of the system when the end state is exactly at the phase transition. We end in section 5 with a summary and an outlook.

\section{Setup}

\subsection{Action and equations of motion}

We consider the bottom-up model proposed in [8]. The bulk spacetime is that of a $(2+1)$ dimensional finite temperature BTZ black brane,

$$
d s^{2}=G_{\mu \nu} \mathrm{d} x^{\mu} \mathrm{d} x^{\nu}=\frac{L^{2}}{z^{2}}\left(-h(z) \mathrm{d} t^{2}+\frac{\mathrm{d} z^{2}}{h(z)}+\mathrm{d} x^{2}\right), \quad h(z)=1-\frac{z^{2}}{z_{H}^{2}},
$$

where $L$ is the $A d S_{3}$ radius, and $z$ is the radial coordinate, with the boundary at $z=0$ and the horizon at $z=z_{H}$. The temperature of the dual field theory corresponds to the black brane's Hawking temperature, $T=1 /\left(2 \pi z_{H}\right)$. We apply the scaling symmetries available to set $z_{H}=1$ and $L=1$ for the rest of this work. 
The model of [8] has a non-dynamical co-dimension one hypersurface at $x=0$, which provides the gravity dual of the localised Kondo impurity of the boundary field theory. The action consists of an $A d S_{3}$ bulk contribution involving the Chern-Simons gauge field $A$, and an $A d S_{2}$ defect contribution involving a complex scalar $\Phi$ and a $\mathrm{U}(1)$ gauge field $a,{ }^{1}$

$$
\begin{aligned}
S & =S_{\mathrm{CS}}+S_{\mathrm{AdS}_{2}}, \\
S_{\mathrm{CS}} & =-\frac{N}{4 \pi} \int \operatorname{tr}\left(A \wedge d A+\frac{2}{3} A \wedge A \wedge A\right), \\
S_{\mathrm{AdS}_{2}} & =-N \int \mathrm{d}^{2} x \sqrt{-g}\left[\frac{1}{4} f^{m n} f_{m n}+g^{m n}\left(D_{m} \Phi\right)^{\dagger} D_{n} \Phi+V\left(\Phi^{\dagger} \Phi\right)\right],
\end{aligned}
$$

where $f_{m n}=\partial_{m} a_{n}-\partial_{n} a_{m}$ is the $\mathrm{U}(1)$ field strength, $D_{m}$ is the gauge-covariant derivative,

$$
D_{m} \Phi=\left(\partial_{m}+i A_{m}-i a_{m}\right) \Phi
$$

and the $A d S_{2}$ metric $g$ is the pullback of the $A d S_{3}$ metric $G$ to the hypersurface by the immersion $x=0$. The Roman indices $m, n$ run over $t, z$. For the remainder of this paper, we restrict to a U(1) flavour symmetry and thus the Chern-Simons contribution (2.3) to the action becomes Abelian. The equations of motion are [8]

$$
\begin{aligned}
\epsilon^{n \mu \nu} F_{\mu \nu} & =\frac{4 \pi}{N} \delta(x) J^{n}, \\
\partial_{m}\left(\sqrt{-g} g^{m p} g^{n q} f_{p q}\right) & =J^{n}, \\
\partial_{n} J^{n} & =0, \\
\partial_{m}\left(\sqrt{-g} g^{m n} \partial_{n} \phi\right) & =\sqrt{-g} \Delta^{m} \Delta_{m} \phi+\frac{1}{2} \sqrt{-g} \frac{\partial V}{\partial \phi},
\end{aligned}
$$

where we parametrised $\Phi=\phi e^{i \psi}$ and defined

$$
J^{n} \equiv 2 \sqrt{-g} g^{m n} \Delta_{m} \phi^{2}, \quad \Delta_{m} \equiv a_{m}-A_{m}-\partial_{m} \psi,
$$

where greek indices run from 0 to 2 and latin indices from 0 to $1 . A_{m}$ are understood to be the components of the projection of $A$ to the hypersurface. Upon gauging $A_{z}=0$ and requiring regularity of the CS field at the horizon, $A_{t}\left(z_{H}\right)=0$, only $A_{x}$ remains nontrivial. Hence, the projection of the Chern-Simons field to the defect hypersurface vanishes which implies that $A$ decouples from the rest of the fields. Due to this we are allowed to neglect the CS field when solving for the fields restricted to the defect. In principle, the CS field could be integrated from the solutions of $\Phi$ and $a$.

The field content of the model defined by equations (2.2)-(2.4) is to be interpreted in the light of the holographic dictionary outlined in $[8,16]$. Specifically, the Chern-Simons gauge field $A$ is holographically dual to the chiral current of conduction electons $\psi$ in the boundary theory. Similarly, the gauge field $a$, which is restricted to the $A d S_{2}$ subspace at $x=0$, is dual to the charge of the slave fermions $\chi$ that are restricted to the impurity at

\footnotetext{
${ }^{1} \mathrm{In}[16]$, a $\mathrm{U}(2)$ gauge group was assumed for the gauge field $a$, corresponding to a two impurity Kondomodel.
} 
$x=0$ in the field theory picture. The scalar $\Phi$, charged under both the gauge groups of the fields $a$ and $A$, is then dual to the composite operator $\mathcal{O}=\psi^{\dagger} \chi$, which indicates the coupling of the conduction band to the impurity. The potential term $V\left(\Phi^{\dagger} \Phi\right)$ in $(2.4)$ is chosen as

$$
V\left(\Phi^{\dagger} \Phi\right)=M^{2} \Phi^{\dagger} \Phi
$$

with $M^{2}$ tuned to the Breitenlohner-Freedman bound to obtain the correct scaling dimensions. At temperatures below a critical one, $T_{c}$, the scalar field exhibits an instability, which leads to its condensation and thus a nonvanishing expectation value, $\langle\mathcal{O}\rangle \neq 0$. This is interpreted as the formation of the Kondo cloud in the large- $N$ holographic model we consider here. We shall refer to the phase above $T_{c}$ where the scalar does not condense as the normal phase, and the phase below $T_{c}$ where it does as the condensed phase. In the condensed phase, the impurity is screened. Further details may be found in $[8,16]$. Below, we review only those previous results that are relevant in the context of this paper. We present our new results on time dependence in sections 3 and 4.

\subsection{Boundary behaviour and conditions on the Kondo coupling}

In the normal phase $T>T_{c}$, the solution for the gauge field is given by

$$
a_{t}(t, z)=\frac{Q}{z}+\mu
$$

where $Q$ denotes the electric flux at the boundary and $\mu$ the chemical potential. As explained in [8], $Q$ defines the representation of the impurity spin and by following the same conventions, we set $Q=-\frac{1}{2}$. $|Q|$ is related to the number of boxes $q$ in the spin representation Young tableau by $|Q|=q / N$. In order to obtain regularity at the horizon, we must set $\mu=-Q$. To be able to map our bottom-up model to the Kondo model, we need to fix the scaling dimension of the scalar operator to be $\Delta_{\mathcal{O}}=\frac{1}{2}$, see [8]. As the scalar field is restricted to live in an asymptotically $A d S_{2}$ space, the scaling dimension is given by

$$
\Delta_{\mathcal{O}}=\frac{d}{2} \pm \sqrt{\frac{d^{2}}{4}-Q^{2}+M^{2}}
$$

where we set $d=1$ [25]. It can be seen that the correct scaling dimensions can only be obtained if we put the scalar exactly at the Breitenlohner-Freedman bound. With our choice of $Q$, this means setting $M=0$. The leading order behaviour of the scalar field near the bounday is then given by

$$
\Phi(t, z)=\phi_{1}(t, z)+i \phi_{2}(t, z) \approx \sqrt{z}(\alpha(t) \log (z)+\beta(t))+\ldots
$$

where $\alpha(t)=\alpha_{1}(t)+i \alpha_{2}(t)$ and $\beta(t)=\beta_{1}(t)+i \beta_{2}(t)$ are complex functions of time. As was shown in $[8,12,16]$, the boundary condition for a boundary double trace operator $\sim \kappa \mathcal{O O}^{\dagger}$ is given by requiring $\alpha=\kappa \beta$. Furthermore, it was demonstrated that an arbitrary energy scale $\Lambda$ has to be introduced due to the appearence of the logarithm in the boundary expansion. The Kondo coupling $\kappa$ is running w.r.t. rescalings of $\Lambda$ and eventually diverges at low temperatures at the Kondo temperature $T_{K}=\Lambda e^{1 / \kappa} / 2 \pi$, which is invariant under 
these rescalings. We avoided this energy scale by rescaling $z$ by $z_{H}$, which renders the radial coordinate $z$ dimensionless. To avoid confusion, note that the notation of $\kappa, \beta, \alpha$ in [8] differs from ours by a subscript $(\cdot)_{T}$, which we left out for convenience.

We impose a time-dependent boundary condition on the Kondo coupling $\kappa(t)=\kappa_{1}(t)+$ $i \kappa_{2}(t)$ which is a real function of time, i.e. we set $\kappa_{2}(t)=0$. The relationships between the leading and subleading expansion coefficients at the boundary are hence given by

$$
\alpha_{1}(t)=\kappa_{1}(t) \beta_{1}(t) \quad \text { and } \quad \alpha_{2}(t)=\kappa_{1}(t) \beta_{2}(t) .
$$

At the same time, the electric flux $Q$ of the gauge field $a$ is required to stay constant, which is actually necessary to render the variational problem meaningful, cf. [18]. Together with regularity at the event horizon, this fixes all boundary conditions for our system of partial differential equations. After going through the holographic renormalisation procedure, which was carefully constructed and carried out in [16], one finds ${ }^{2}$

$$
\langle\mathcal{O}\rangle=-N \beta^{\dagger} .
$$

Since we are interested in the real-time dynamics of the scalar operator, $\beta$ will thus be the main quantity we focus on below.

In equilibrium, the temperature measured in units of the Kondo temperature $T_{K}$ can be found from $\kappa_{1}$, and it is the same relationship as that given in [8]:

$$
\frac{T}{T_{K}}=\exp \left(-1 / \kappa_{1}\right)
$$

Furthermore, we have from [18]

$$
\log \frac{T_{c}}{T_{K}}=-2 \operatorname{Re}\left[H\left(-\frac{1}{2}+i Q\right)\right]-\log 2,
$$

where $H(z)$ is the harmonic number. For $|Q|=1 / 2$, which is used throughout this paper, we have

$$
\kappa_{c}=\kappa_{1}\left(T_{c}\right)=\left(2 \operatorname{Re}\left[H\left(\frac{i-1}{2}\right)\right]+\log 2\right)^{-1} \approx 8.9796 .
$$

To study how the system evolves given a Kondo coupling that is changing in time, we investigate the time evolution of the expectation value of the scalar operator, $\langle\mathcal{O}(t)\rangle$. We consider time-dependent profiles for the Kondo coupling with a form of either a hyperbolic tangent,

$$
\kappa_{1}(t)=\kappa_{i}+\frac{\Delta \kappa}{2}\left[\tanh \left(s\left(t-t_{0}\right)\right)+1\right],
$$

or a Gaussian,

$$
\kappa_{1}(t)=\kappa_{i}+\Delta \kappa \exp \left(-s\left(t-t_{0}\right)^{2}\right) .
$$

We consider the system to be initially prepared in an equilibrium state characterised by the Kondo coupling $\kappa_{i}$, which is then quenched to another state whose equilibrium is

\footnotetext{
${ }^{2}$ In [8], the expectation value of the dual scalar operator was identified as $\langle\mathcal{O}\rangle \propto \alpha$. See [16] for a complete discussion of the difference.
} 
characterised by $\kappa_{f}$. For a hyperbolic tangent quench, $\kappa_{f}=\kappa_{i}+\Delta \kappa$, whereas for a Gaussian quench, $\kappa_{f}=\kappa_{i}$. In both cases, the amplitude parameter $\Delta \kappa$ controls how much $\kappa_{1}$ can change during the quench. The steepness parameter $s$ controls the speed of the quench, while the offset $t_{0}$ determines the midpoint of the quenching process. We shall refer to the set of parameters $\left\{\kappa_{i}, \kappa_{f}, \Delta \kappa, s, t_{0}\right\}$ as the quench parameters.

In view of the numerical analysis, we change to Eddington-Finkelstein coordinates and choose the radial gauge for the defect gauge field $a$. The numerical solution of the evolution involves pseudospectral methods in the radial direction and an implicit evolution method in the time direction. More details of the numerical implementation are given in appendices A and B.

\section{Results}

\subsection{Generic time evolution for phase transitions}

Here we present examples which show the generic form of the time-evolution of the scalar condensate as the system is quenched from one phase to a different one. The input to the time-evolution problem is $\kappa_{1}(t)$, which we choose to have a hyperbolic tangent profile as given in (2.20). Similar quenches of a double-trace coupling were studied in [24], but in a different setting without defect. Given the quench profile of the Kondo coupling, we solve the equations of motion to obtain the evolution of $\beta_{1,2}(t)$ and $\mu(t)$, and extract from it information about the condensate $\langle\mathcal{O}(t)\rangle$. As we explain below, QNMs can also be extracted from the time-evolution of the scalar condensate.

Figures 1 to 3 show the time evolution of the scalar operator expectation value $\langle\mathcal{O}(t)\rangle$ for a quench from the normal phase $\left(T>T_{c}, \kappa_{1}>\kappa_{c}\right)$ to the condensed phase $\left(T<T_{c}\right.$, $\left.\kappa_{1}<\kappa_{c}\right)$. The profile for this "normal-to-condensed" quench, $\kappa_{1}^{n c}(t)$, is shown in figure 1 (a). Figure 1 (b) shows the absolute value of $\langle\mathcal{O}(t)\rangle$, and we see first a clear exponential rise (figure $1(\mathrm{c})$ ), and then an exponential decay to a constant value (figure $1(\mathrm{~d})$ ). ${ }^{3}$ Figure 2 shows finer details of the time evolution for the normal-to-condensed quench, $\kappa_{1}^{n c}(t)$. We see that $\langle\mathcal{O}(t)\rangle$ oscillates and then settles exponentially to a non-zero value dictated by $\kappa_{f}$ in the condensed phase. Note that appreciable changes in $\langle\mathcal{O}(t)\rangle$ do not begin until well after the end of the quench.

We model the time evolution of the scalar field using a QNM behaviour of the form

$$
f(t)=a e^{-i \omega t}+b, \quad \omega=\omega_{R}+i \omega_{I},
$$

to fit our results, with $\omega$ is the complex QNM frequency. The fit is depicted by the red curve in figure 2 , which agrees very well with the full numerical result given by the blue curve. The initial behaviour just after the quench is described by a QNM with $\omega_{R} \neq 0$, leading to an oscillation profile, and with $\omega_{I}>0$, leading to an exponential rise indicating

\footnotetext{
${ }^{3}$ In the normal phase, the scalar condensate vanishes, $\langle\mathcal{O}\rangle \propto \beta^{\dagger}=0$. However numerically, zero is only represented up to machine precision, i.e. a finite quantity is regarded as zero if its magnitude is less than the machine precision. To avoid numerical artefacts at machine precision and to have a firmer control of the numerical accuracy, we set $\beta_{1}(t=0)=\beta_{2}(t=0)=10^{-10}$, which explains the initial plateau.
} 


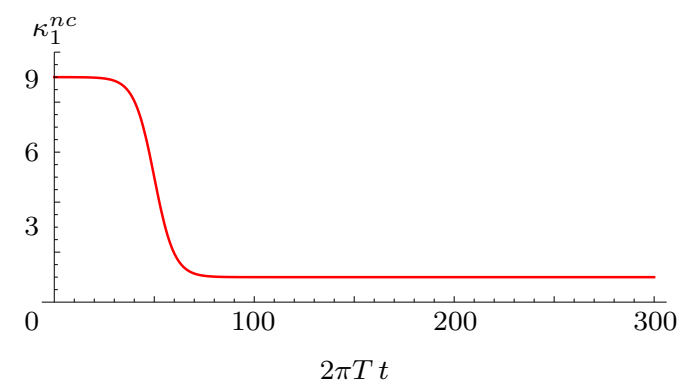

(a) Quench profile for $\kappa_{1}$ as given by eq. (2.20) with parameters $\kappa_{i}=9, \kappa_{f}=1, s=1 / 10$, and $2 \pi T t_{0}=50$.

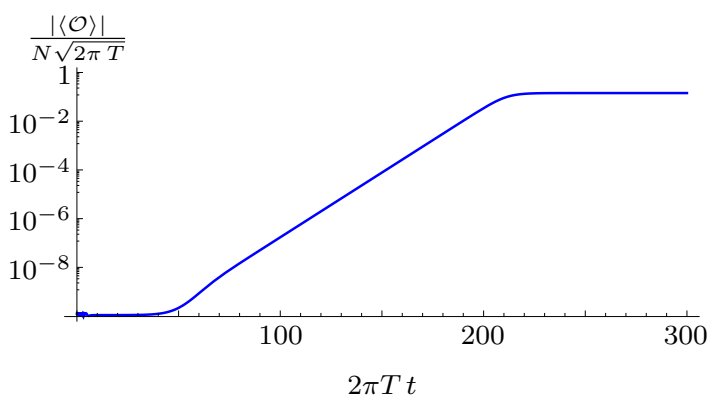

(c) Log plot of (b), indicating the exponential instability.



(b) Time evolution of the absolute value of $\langle\mathcal{O}\rangle$.

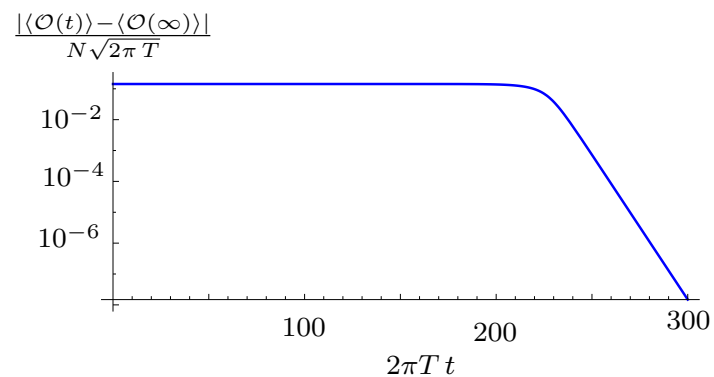

(d) Log plot for the deviation at late times, indicating the QNM ringdown to the equilibrium value for the new coupling $\kappa_{f}=\kappa_{1}^{n c}(\infty)$.

Figure 1. Typical numerical evolution of the scalar operator (b) for a quench from the normal to the condensed phase (a). Note the different time scales involved due to the instability mode (c) and the QNM ringdown to the final equilibrium (d).



(a)



(b)

Figure 2. Time evolution of the real and imaginary part of $\langle\mathcal{O}\rangle$ (a), and the normalised absolute value of $\operatorname{Re}\langle\mathcal{O}\rangle$ (b) for the normal-to-condensed quench, $\kappa_{1}^{n c}(t)$. The red curve in (b) is a numerical fit to the unstable QNM behaviour of the form given by eq. (3.1), with $\omega_{I}>0$.

an instability. Physically, this comes about since the quench is driving the system out of the normal phase, and instabilities must occur prior to the formation of a scalar condensate leading to the new stable ground state. Just after the quench, the dominant QNM is thus associated with the instability of the normal phase. We will make this more precise in the next subsection. 


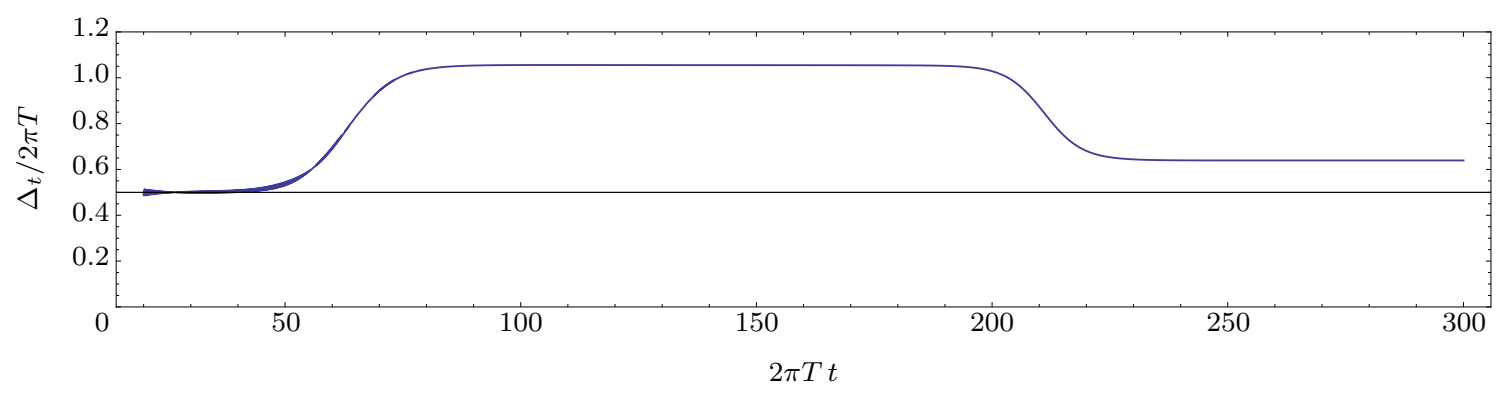

Figure 3. Time evolution of $\Delta_{t}=\mu-\partial_{t} \psi_{0}$ for the normal-to-condensed quench $\kappa_{1}^{n c}(t)$.

On the other hand, the relaxation at late times is described by a QNM with $\omega_{I}<0$ and $\omega_{R}=0$, i.e. a pure exponential decay. Such behaviour is expected as the system reach near its final equilibrium state, and is controlled by the QNM of the final state, see e.g. [26]. Note that this exponential decay is governed by the lowest-lying QNM, i.e. one with the smallest $\left|\omega_{I}\right|$. Higher QNMs would produce much faster decays than that from the lowest QNM, which would be exponentially suppressed in comparison, and not be visible at late times. Note also that since the time-evolution is described by the QNMs, its time scales are given by the appropriate $\omega_{I}^{-1}$ in the appropriate regime. These scales are all long compared to the time scale of the quench profile.

Figure 3 shows the time evolution of the gauge invariant quantity $\Delta_{t}=\mu-\partial_{t} \psi_{0},{ }^{4}$ for the normal-to-condensed quench $\kappa_{1}^{n c}(t)$. It starts out at $\approx 1 / 2$ since in the normal phase $\mu(0)=1 / 2$ and the phase rotation $\partial_{t} \psi_{0}$ is approximately zero if the coupling parameter $\kappa_{i}=9$ is close to its critical value. As the system is quenched, $\Delta_{t}$ rises to an intermediate plateau. This is due to the fact that an instability mode of the normal phase is turned on, whose imaginary part is just the phase rotation velocity. Eventually at $2 \pi T t \approx 200$, the scalar field becomes macroscopic in size, see figure $1 \mathrm{~b}$. This causes backreaction on $\Delta_{t}$, which then drops to a new asymptotic constant value for $2 \pi T t>200$.

Let us now turn to a quench from the condensed to the normal phase, as shown in figure 4 to 6 . The profile for the condensed-to-normal quench, $\kappa_{1}^{c n}(t)$, is shown in figure 4a. Figure $4 \mathrm{~b}$ shows the absolute value of $\langle\mathcal{O}(t)\rangle$, and we see now a clear exponential decay to zero right after the quench, as expected for the scalar in the normal phase.

Figure 5 shows finer details of the time evolution for the condensed-to-normal quench, $\kappa_{1}^{c n}(t)$. We see that $\langle\mathcal{O}(t)\rangle$ oscillates and then settles exponentially to zero in the normal phase. Again, the time evolution is very well described by the QNM behaviour of the form given by eq. (3.1). This time, the governing QNM is the lowest-lying in the normal phase with $\omega_{I}<0$. Note the period of the oscillations is much longer (by one to two orders of magnitude) compared to the normal-to-condensed quench since here, $\left|\omega_{I}\right|$ is much smaller.

Interestingly, the QNM behaviour takes over almost immediately after the quench, rather than at late times. There does not appear to be a nonlinear regime between the end of the quench and the start of the ringdown. This appears to be a universal feature of holography, where strongly-coupled systems are modelled by dual gravitational dynamics [26, 27].

\footnotetext{
${ }^{4}$ The quantity $\psi_{0}$ is the leading order expansion coefficient of the phase of the scalar field, and is given by $\psi_{0}=\arctan \left(\beta_{2}(t) / \beta_{1}(t)\right)$.
} 


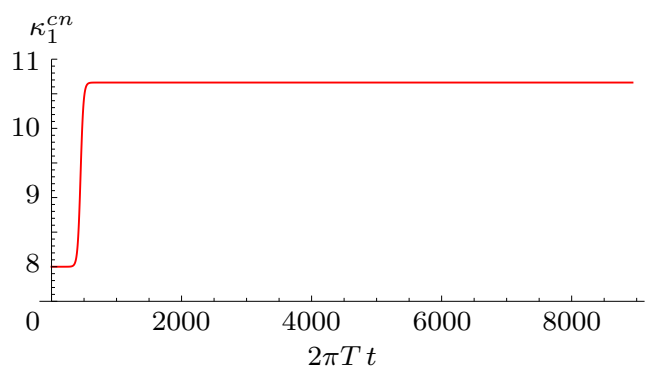

(a)



(b)

Figure 4. The quench profile (left) and the time evolution of the absolute value of $\langle\mathcal{O}\rangle$ (right) for a quench from the condensed to the normal phase. The quench parameters are $\kappa_{i}=8, \kappa_{f} \approx 10.7$, $s \approx 0.022$ and $2 \pi T t_{0} \approx 447$.
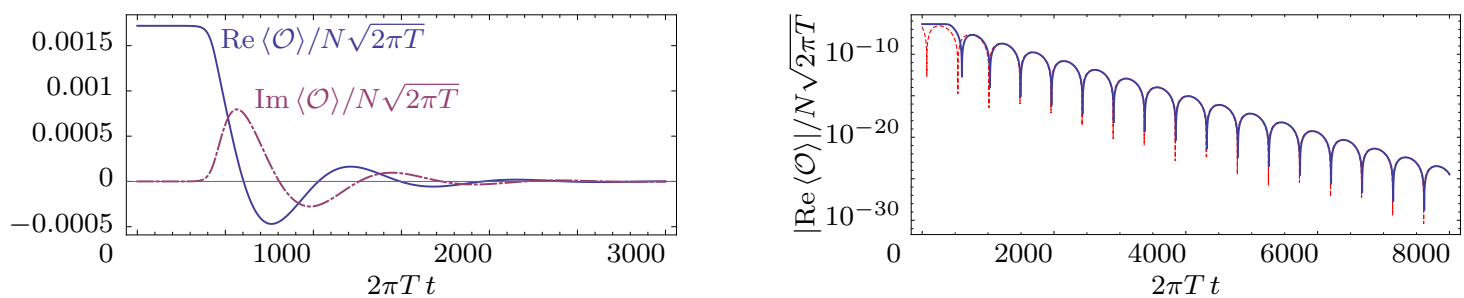

Figure 5. Time evolution of the real and imaginary part of $\langle\mathcal{O}\rangle$ (left), and the absolute value of $\operatorname{Re}\langle\mathcal{O}\rangle$ (right) for the condensed-to-normal quench, $\kappa_{1}^{c n}(t)$. The red curve in the right plot is a numerical fit to the lowest QNM ringdown of the form given by eq. (3.1).



Figure 6. Time evolution of the gauge invariant quantity, $\Delta_{t}=\mu-\partial_{t} \psi_{0}$.

Figure 6 shows the time evolution of the gauge invariant quantity $\Delta_{t}=\mu-\partial_{t} \psi_{0}$ for the condensed-to-normal quench $\kappa_{1}^{c n}(t)$. Compared to the normal-to-condensed quench, there is no plateau or basin structure seen between the initial and the final equilibrium values of $\Delta_{t}$. The value of $\Delta_{t}$ does not asymptote to $\mu_{c}=1 / 2$ (for $\left.Q=1 / 2\right)$ due to the ongoing phase rotation of the scalar field, whose velocity asymptotes to a constant which matches the offset in 6. This offset arises from the real part of the lowest QNM of the normal phase. 


\subsection{QNMs in normal phase}

By the holographic correspondence, the QNMs of the scalar fluctuations are related to the poles of the two-point function of the dual scalar operator $\mathcal{O}$. In the normal phase, these poles are analyically given by $[17]$

$$
\frac{1}{\kappa_{T}(\omega)}=-\log \frac{T}{T_{K}}=H\left(-\frac{1}{2}+i Q-i \frac{\omega}{2 \pi T}\right)+H\left(-\frac{1}{2}-i Q\right)+\log 2 .
$$

For complex frequencies, $\kappa_{T}(\omega)$ is a complex function. As the temperature is lowered, poles in the lower half of the complex $\omega$-plane move up towards the origin, arriving there at the critical temperature, $T_{c}$. They cross into the upper half plane below $T_{c}$, signalling an instability [17]. This is qualitatively similar to the behaviour of the lowest-lying QNMs in holographic superconductors $[7,28]$. The critical coupling at which phase transition occurs is thus $\kappa_{T}(0)=\kappa_{c}$, the same critical value as that given in eq. (2.19).

Figure 7 shows a contour plot of the magnitude of the Kondo coupling, $|\kappa|$, on the complex $\omega$ plane. The dashed curve traces out a path on which $\kappa$ is real. Following this curve, we have $\kappa>\kappa_{c}\left(T>T_{c}\right)$ in the lower half plane, $\kappa<\kappa_{c}\left(T<T_{c}\right)$ in the upper half plane, and $\kappa=\kappa_{c}$ at the origin as expected. The right of figure 7 shows a blow-up of the region in the contour plot around the origin marked by the red square. The QNMs found numerically from fitting the time evolution of the scalar condensate to the QNM behaviour given by eq. (3.1) are marked by the red dots. Those coming from fitting the exponential rise in the normal-to-condensed quench give QNMs in the upper half plane, while those from the exponential decay in the condensed-to-normal quench give QNMs in the lower half plane. We see that they fall perfectly on the dashed curved analytically given by eq. (3.2), indicating that it is indeed the normal phase QNM associated with instability that governs the exponential rise seen in figure 1, and the lowest QNM in the normal phase that governs the relaxation seen in figure 4.

Note that close to $T_{c}$, QNMs in the normal phase can be found analytically [18]. In particular, the lowest QNM is given by

$$
\frac{\omega}{2 \pi T_{c}}=\frac{-i}{\psi^{\prime}\left(\frac{1}{2}+i Q\right)}\left(\frac{T}{T_{c}}-1\right)
$$

which implies a relaxation time scale

$$
\tau \sim \omega^{-1} \sim \frac{1}{T_{K}}\left(\frac{T}{T_{c}}-1\right)^{-1}, \quad T \gtrsim T_{c}
$$

and we have used the fact that $T_{c} \sim T_{K}$ for $|Q|=1 / 2$.

\subsection{QNMs in condensed phase}

In the condensed phase, the two-point function of the scalar operator, as well as the associated QNMs, can be found by solving a coupled system of fluctuation equations. Close to $T_{c}$ when the scalar condensate is small, the QNMs can also be found semi-analyically, see [18]. Here, we obtain QNMs in the condensed phase in yet another way by fitting the relaxation of $\langle\mathcal{O}\rangle$ after Gaussian quenches, and we find excellent agreement to those found in [18]. 


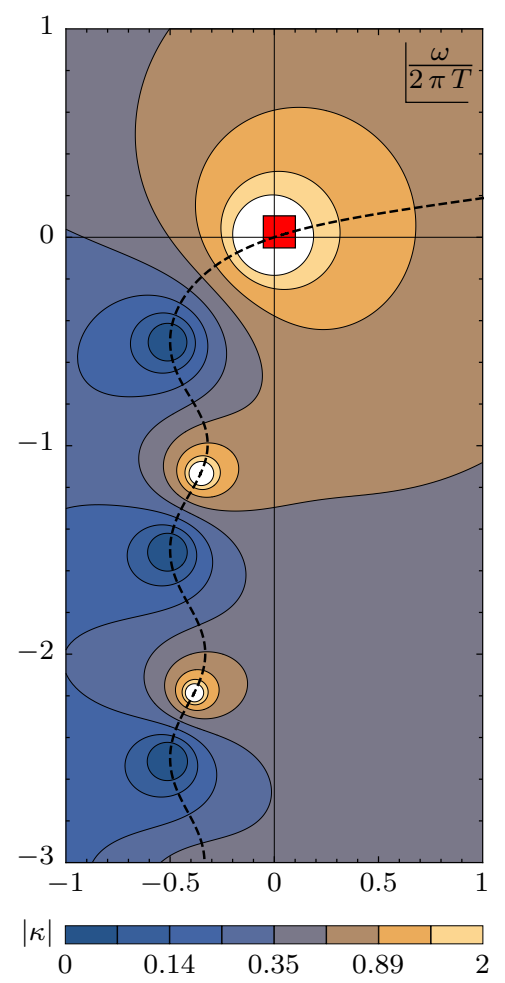

(a) Contour plot of $|\kappa|$ in the complex frequency plane. White space denotes cropping for values $|\kappa|>2$.

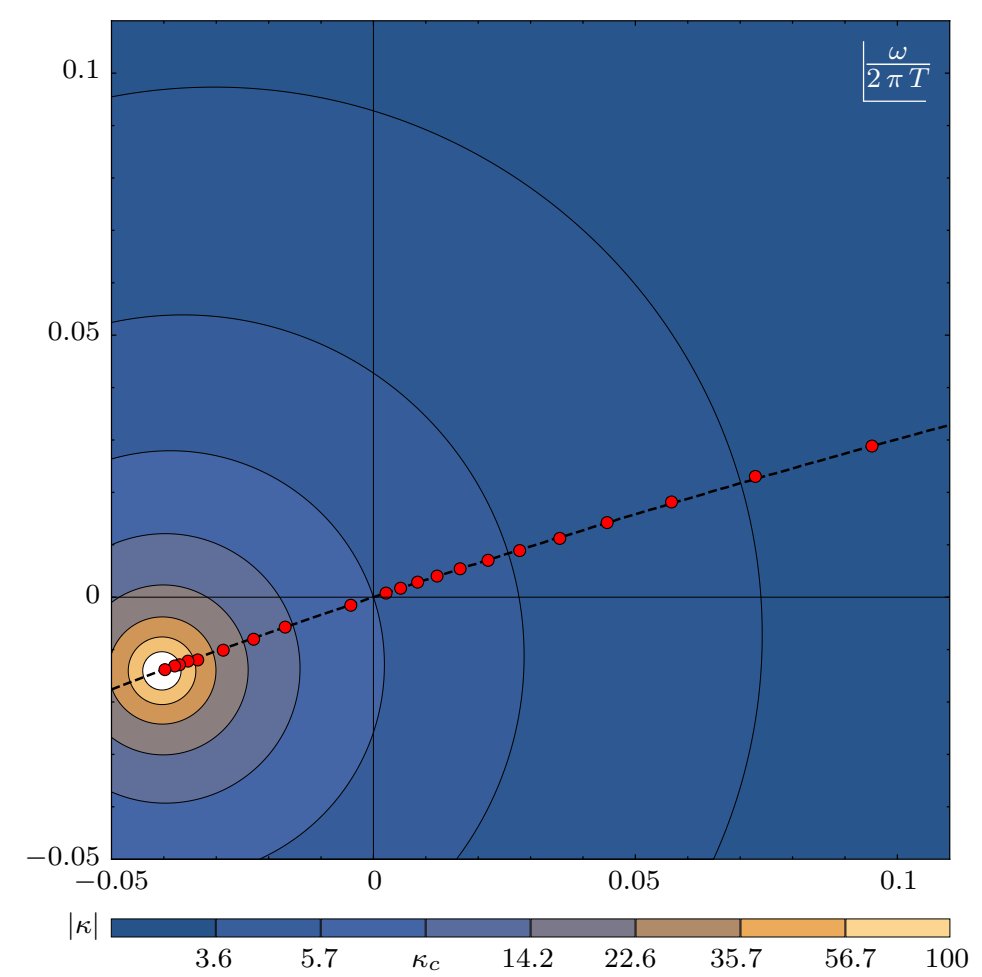

(b) Blowup of the red square in (a). Red dots indicate QNMs extracted from numerical fitting at different temperatures. White space denotes cropping for values $|\kappa|>100$.

Figure 7. Contour plot of $|\kappa(\omega)|$ over the complex $\omega$-plane. A blow-up around the origin is shown in (b). Along the dashed curve $\kappa(\omega)$ is real. The contours denote constant values of $|\kappa|$, with the colour scale indicating the value. The contour $\kappa(\omega)=\kappa_{c}$ intersects the dashed line at the origin in (b). The red dots are lowest lying QNM found from fitting the time evolution of the scalar operator after the quench to the QNM behaviour defined in eq. (3.1).

Figure 8a shows the lowest QNMs, i.e. QNMs closest to the real axis, found from fitting the relaxation at late times after a quench from states initially in the condensed phase at various $T<T_{c}$. We see they are all purely imaginary, and move down the imaginary axis as the temperature is lowered, all in agreement with [18]; the relaxation of the condensate is then a pure exponential decay without oscillations. Figure $8 \mathrm{~b}$ exhibits the temperature dependence of the lowest QNM in the condensed phase, when the temperature is normalised by $T_{c}$. We see that this purely imaginary mode first moves down the imaginary axis as temperature decreases below $T_{c}$ and then turnes back up towards the origin. This shows that the system experiences critical slowing down both as $T \rightarrow T_{c}$ and as $T \rightarrow 0$ (see section 4.1 for further discussion of this phenomenon in the $T \rightarrow T_{c}$ limit).

The over-damped relaxation behaviour has been seen before in $[6,7]$, which performed the quench differently and employed a different quench protocol. ${ }^{5}$ In particular, in [7] the

\footnotetext{
${ }^{5}$ The quench were enacted through the source of the dual scalar operator, and Gaussian quench protocol were used.
} 




(a) Normalised to $2 \pi T$ and shown in complex plane.

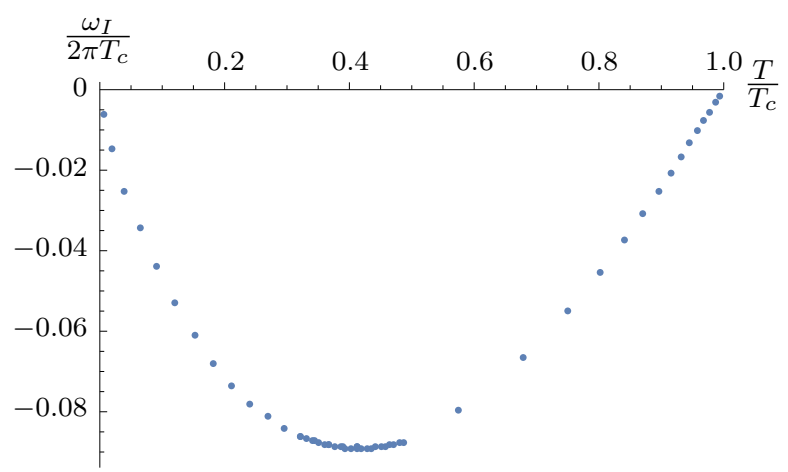

(b) Normalised to $2 \pi T_{c}$ and plotted vs. $T / T_{c}$.

Figure 8. The lowest QNMs extracted from fitting the relaxation behaviour after Gaussian quenches in the condensed phase characterised by $0.008 \leq T / T_{c} \leq 1$.

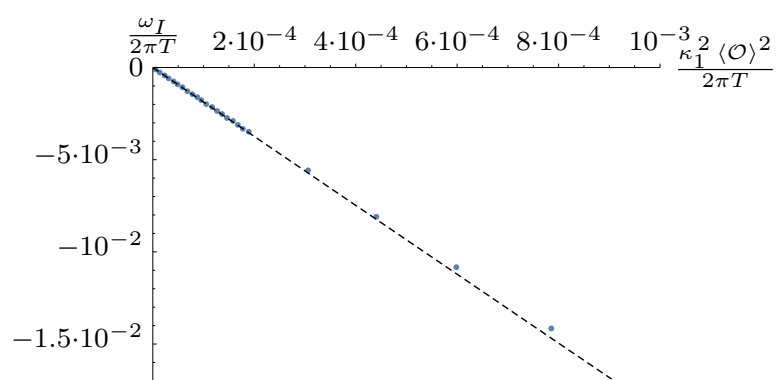

(a) Behaviour for $T \lesssim T_{c}$. The dashed line is a linear fit for the region $1>T / T_{c}>0.998$ passing through the origin.



(b) Full temperature range from $T / T_{c}=1$ down to $T / T_{c} \approx 0.008$. The dashed line is a fit for the region $T / T_{c}<0.33$.

Figure 9. Functional dependence of the lowest QNM on $\kappa_{1}^{2}\langle\mathcal{O}\rangle^{2}$ in the condensed phase.

lowest (most dominant) QNMs that encode the relaxation at late times were also seen to move down the imaginary axis as temperature were lowered below $T_{c}$. However in [7], a regime of damped oscillatory relaxation were found for some $T_{*}<T_{c}$, at which point the previously sub-dominant QNMs become the dominant ones. We note that backreaction is icluded in [7]. For our kind of quench, we have not found such damped oscillatory regime within the temperature range where the probe approximation is valid. To explore whether such regime exists for our quench in our system at even lower temperatures would require the inclusion of backreaction, which is beyond the scope of the current work.

Figure 9 shows how the lowest QNM vary as a function of $\kappa_{1}^{2}\langle\mathcal{O}\rangle^{2}$. Close to $T_{c}$, we find that the lowest QNM has a linear dependence on $\kappa_{1}^{2}\langle\mathcal{O}\rangle^{2} / N^{2}$,

$$
\omega_{I}=b \kappa_{1}^{2}\langle\mathcal{O}\rangle^{2} / N^{2}, \quad b \approx-18.7,
$$

where $b$ is found from a numerical fit. This value of $b$ is very close to that in [18]. The difference is about $6 \%$, and is due to the slight differences in the data being fitted. Deviation from this linear relation began to be visible for $T>0.988 T_{c}$. Note that from [8], $\langle\mathcal{O}\rangle \propto$ 
$\left(T_{c}-T\right)^{1 / 2}$ for $T \lesssim T_{c}$. Given $T / T_{K}=e^{-1 / \kappa_{1}}$, this implies a relaxation time scale

$$
\tau \sim \omega^{-1} \sim \frac{b}{T_{K}} \log ^{2} \frac{T}{T_{K}}\left(1-\frac{T}{T_{c}}\right)^{-1}, \quad T \lesssim T_{c} .
$$

Interestingly, at low temperatures we observe a switching to a logarithmic dependence from the linear one found close to $T_{c}$ :

$$
\frac{\omega_{I}}{2 \pi T}=b \log \left(\frac{\kappa_{1}^{2}\langle\mathcal{O}\rangle^{2}}{2 \pi T N^{2}}\right), \quad b \approx-0.14 .
$$

Such log behaviour would indicate a deviation from mean-field behaviour. To understand the origin of this $\log$ behaviour and be sure that it persists all the way down to $T=0$ requires a study of the IR behaviour around zero temperature. This requires, however, the inclusion of full backreaction and stabilisation of the scalar at the IR fixed point ${ }^{6}$ which is beyond the scope of the current work. We plan to address these questions in the future, which are also interesting in the context of finding the zero-temperature impurity entropy at the defect [14].

\subsection{Evolution of the screening of the impurity}

One particular goal of the analysis presented is to obtain the time evolution of the screening due to the formation of the Kondo cloud. According to [8], the electric flux at the asymptotic boundary and the event horizon provides a measure of the number of impurity degrees of freedom in the UV and the IR, respectively. Its decrease thus corresponds to the screening of the impurity in the IR. This is due to the fact that the flux involves $a_{t}$, which is dual to the charge density determining the size of the $\mathrm{SU}(N)$ spin representation. The electric flux at the event horizon is a quantity that may be traced in Eddington-Finkelstein coordinates. Although there is no straightforward map of horizon dynamics to the boundary, the decay constant of the horizon flux still encodes information about the decrease of impurity degrees of freedom.

In our choice of coordinates and gauge fixing (see appendix A), $a_{v}^{\prime}(v, y)$ is proportional to the electric flux. We define a new variable $D$ by

$$
D=\frac{a_{v}^{\prime}(v, 1)}{a_{v}^{\prime}(0,1)}-1
$$

which starts out at zero at $t=0$ by construction and becomes nontrivial during a generic quench. In figure 10, we show the evolution of $D$ for a Gaussian quench around $\kappa_{1}=1$ and $\kappa_{1}=8.5$, respectively. We observe an initial rise due to the Gaussian quench which takes the system to a state with smaller condensate, and subsequently an exponential decay which corresponds to the reduction of impurity degrees of freedom due to screening. For the Kondo coupling $\kappa_{1}=8.5$ shown in figure $10 \mathrm{~b}$, which is closer to the phase transition, there is a plateau at intermediate times which is a sign of the onset of critical slowingdown near the phase transition. Fitting the evolution at late times to an exponential decay

\footnotetext{
${ }^{6}$ This can be done, for example, by adding a quartic term to the scalar potential in (2.11).
} 


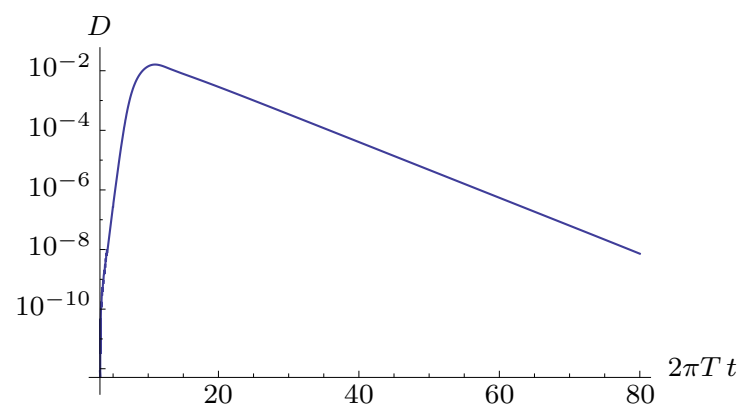

(a) $\kappa_{1}=1$, i.e. $T \approx 0.41 T_{c}$.

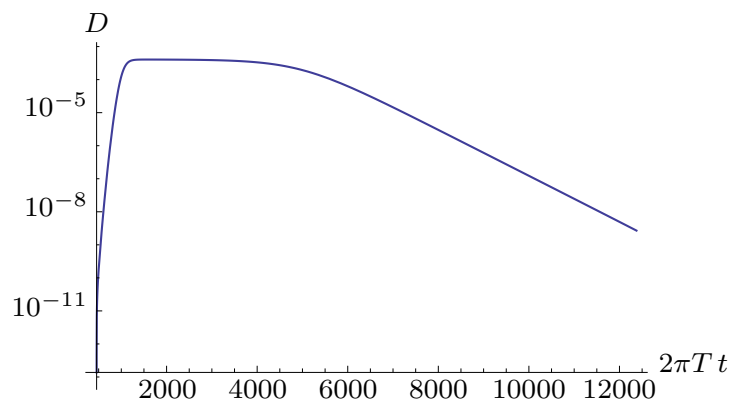

(b) $\kappa_{1}=8.5$, i.e. $T \approx 0.99 T_{c}$.

Figure 10. A log plot of $D$ as defined in (3.8) for different Gaussian quenches, showing the latetime exponential decay of the effective IR degrees of freedom due to screening. Signs of critical slowing down can be seen in (b) as the system is close to the critical temperature $T_{c}$.

reveals that it is governed by a QNM which, not suprisingly, coincides with the complex frequency obtained by analysing the time dependence of boundary quantities. Of course, it would be interesting to examine further observables that contain information about the evolution of the screening, in particular the evolution of the Kondo cloud in the spatial direction ambient to the defect (i.e. in $1+1$ dimensions). This will allow a comparison to the Kondo cloud evolution obtained e.g. in [23]. This may be obtained holographically with methods proposed in [14], however for time-dependent couplings. It requires to include the backreaction of the geometry to the field content on the defect hypersurface. This is left for future research.

\section{Critical behaviour}

In this section we consider quenches for which the final value of the Kondo coupling $\kappa$ is either very close to or exactly at the critical value $\kappa_{c}$ where the phase transition takes place. In the former case, we find the expected critical slowing down. In the latter case, instead of an exponential decay we observe a polynomial one. In particular, looking at the real and imaginary parts of the condensate separately, we observe a so-called log-periodic oscillation, which is a sign of discrete scale invariance.

\subsection{Critical slowing down}

In subsections 3.2 and 3.3 , we saw that $\omega_{I} \rightarrow 0$ as the phase transition at $\kappa=\kappa_{c}$ is approached. This implies that near the phase transition, the characteristic time scale $\tau=\omega_{I}^{-1}$ diverges, a well known phenomenon known as critical slowing down. Specifically, the theory of dynamic critical phenomena (see e.g. [29] and [30-33] for a holographic context) suggests a divergence of the form

$$
\tau \sim\left(\frac{T-T_{c}}{T_{c}}\right)^{-z \nu}
$$


where $z$ and $\nu$ are critical exponents. For holographic superconductors, it was found that

$$
z=2 \text { and } \nu=\frac{1}{2}
$$

independently of the dimension [31-33]. ${ }^{7}$ As the Kondo model under consideration resembles a holographic superconductor in $A d S_{2}$ space, we expect it to fall into the same universality class, i.e. to have the same critical exponents (4.2). This is indeed the case, as we discuss below. This is non-trivial in the sense that generally, the Coleman-Mermin-Wagner theorem $[35,36]$ precludes phase transitions in low dimensions. However, this theorem does not apply in the large $N$ limit where long-range fluctuations are suppressed [37]. For comparison with higher dimensions we note that also in [24], the exponents (4.2) were found in a holographic model involving a double-trace operator in a background spacetime with black hole horizon.

The holographic Kondo model shows a behaviour of the form (4.1) with an exponent $z \nu=1$, as expected from (4.2). To see this, we begin with the uncondensed phase, where equation (3.2) holds. Linearising this about $\omega=0$, we obtain

$$
\kappa(\omega)=\kappa_{c}+\kappa^{(1)} \omega+\mathcal{O}\left(\omega^{2}\right) .
$$

Here, we have defined the constant

$$
\kappa^{(1)}=\frac{i \psi^{(1)}\left(\frac{1}{2}-\frac{i}{2}\right)}{\left(H_{-\frac{1}{2}+\frac{i}{2}}+\gamma+\log (2)+\psi^{(0)}\left(\frac{1}{2}-\frac{i}{2}\right)\right)^{2}} \approx-189.64+63.20 i,
$$

where $\psi^{(n)}$ is the polygamma function and $\gamma$ is the Euler constant. For complex $\omega$ close to zero and real $\kappa-\kappa_{c}$, we hence see

$$
\tau \sim \omega_{I}^{-1}=\frac{1}{\left(\kappa-\kappa_{c}\right) \operatorname{Im}\left(\frac{1}{\kappa^{(1)}}\right)}
$$

Using $\frac{T}{T_{c}}=e^{\frac{1}{\kappa_{c}}-\frac{1}{\kappa}}$, this yields the result

$$
\tau \sim \frac{1}{\kappa_{c} \operatorname{Im}\left(\frac{1}{\kappa^{(1)}}\right)}\left(\frac{T-T_{c}}{T_{c}}\right)^{-1}
$$

consistent with the expectation based on equations (4.1) and (4.2). This analytic argument described the critical slowing down for $T>T_{c}$, however the critical exponents $z$ and $\nu$ can be defined for both the normal and the condensed phase. In the latter case, where $T<T_{c}$, we do not have analytical results for the relaxation of the system, but as figure 8b shows, our numerical results indicate that at $T \lesssim T_{c}$,

$$
\tau \sim \omega_{I}^{-1} \sim\left(\frac{T-T_{c}}{T_{c}}\right)^{-1}
$$

This again agrees with the expectation from equations (4.1) and (4.2).

\footnotetext{
${ }^{7}$ See also e.g. [34] for a non-holographic study of critical exponents in superconductors.
} 


\subsection{Power-law behaviour and discrete scale invariance at the critical point}

Above we saw that due to the critical slowing down, the time scale governing the exponential decay of perturbations of the system diverges as $\kappa \rightarrow \kappa_{c}$. In the following, we investigate the evolution of the system as we quench the Kondo coupling $\kappa_{1}$ from the condensed phase $\kappa_{1}<\kappa_{c}$ right onto the critical value $\kappa_{1}=\kappa_{c}$.

The numerical results for a quench of this type are shown in figure 11, where we show the time-dependent behaviour of $\beta_{1}(t)$ and $\beta_{2}(t)$ after the quench. We clearly see that the values relax to zero for late times, i.e. that the systems settles to the solution $\Phi=0$ as appropriate for the onset of the normal phase. At first glance, the curves in figure 11 appear to look qualitatively similar to the QNM depicted earlier, e.g. in figure 5. However, there is a significant difference: figure 5 is a log plot and shows a behaviour $\sim \operatorname{Re}\left(e^{-i \omega t}\right)$ with complex $\omega$, while in contrast figure 11 is a log-log plot and hence shows a behaviour

$$
\begin{aligned}
& \beta_{1}(t) \sim \operatorname{Re}\left(e^{-i v \log (t)}\right)=\operatorname{Re}\left(t^{-i v}\right)=t^{v_{I}} \cos \left(v_{R} \log (t)\right), \\
& \beta_{2}(t) \sim \operatorname{Im}\left(e^{-i v \log (t)}\right)=\operatorname{Im}\left(t^{-i v}\right)=-t^{v_{I}} \sin \left(v_{R} \log (t)\right) .
\end{aligned}
$$

Specifically, from the data of figure 11 we may read off

$$
v_{I} \approx-0.502 \text { and } v_{R} \approx 1.51
$$

both of which are less than $1 \%$ off the fractional values of $v_{I}=-1 / 2$ and $v_{R}=3 / 2$. When quenching the system to a final value $\kappa_{\text {final }} \lesssim \kappa_{c}$ or $\kappa_{\text {final }} \gtrsim \kappa_{c}$, the results of the earlier sections 3.2 and 3.3 suggest that we should expect an exponential fall-off with decreasing exponent $\omega$ as $\kappa \rightarrow \kappa_{c}$. Our numerical results show that for $\kappa_{\text {final }}=\kappa_{c}$, the naively expected infinitely slow exponential decay gives way to a power law behaviour of the form (4.8).

In fact, late time power-law tails are common in the study of QNMs. However, they are often associated with the QNMs of asymptotically flat black holes, see e.g. [38] for a review. In asymptotically AdS spaces in contrast, power-law tails of QNMs are usually absent [39]. Furthermore, while in [38] a number of systems are mentioned that exhibit QNMs with power-law tails of the form $t^{-a} \sin (\tilde{\mu} t)$, in (4.8) we observe a so-called $\log$ periodic (damped) oscillation. Here, the amplitude of an oscillating function decays as a power law $\left(\sim t^{-a}\right)$, while the oscillation takes place in logarithmic time $(\sim \sin (b \log t))$. This behaviour is known to be characteristic for systems exhibiting discrete scale invariance and the associated complex critical exponents, see [40] for a review. In short, discrete scale invariance means that a theory is invariant under scale transformations only for specific scales, i.e.

$$
\mathcal{O}_{\mathrm{DSI}}(x)=\hat{\mu} \mathcal{O}_{\mathrm{DSI}}(\lambda x)
$$

only for specific scales $\lambda$. In general, the solution to (4.11) may then take the form [40]

$$
\mathcal{O}_{\mathrm{DSI}}(x) \propto x^{\sigma}, \quad \sigma=-\frac{\log \hat{\mu}}{\log \lambda}+i \frac{2 \pi n}{\log \lambda}
$$


hence the connection with complex exponents and log-periodicity. This phenomenon has been observed ${ }^{8}$ in a wide range of physical systems, including stock markets and earthquakes (see [40] and references therein for these two examples), but also quenches in condensed matter models [41], black hole formation [42-45] and even holographic models [46-48]. See also [49] for a recent application of discrete scale invariance in QFT toy model building. We observe that in our model, similarly to what was found in [44, 45], the discrete scale invariance, i.e. the presence of a non-zero $v_{R}$ in (4.8), manifests itself in form of a phase rotation

$$
\begin{aligned}
t & \rightarrow \lambda t \\
\frac{\alpha(t)}{|\alpha(t)|} & \rightarrow \lambda^{-i v_{R}} \frac{\alpha(t)}{|\alpha(t)|} .
\end{aligned}
$$

The quantities $|\alpha(t)|$ and $|\beta(t)|$ hence do not show any signatures of discrete scale invariance, only a power-law fall-off determined by $v_{I}$. The physical variable in the holographic Kondo model is of course the complex vev $\beta(t) \sim\langle\mathcal{O}\rangle(2.16)$. Hence while the modulus $|\langle\mathcal{O}(t)\rangle|$ decreases as a power-law, its complex phase rotates with $\sim \log t$. Equivalently, we see that the (bulk) gauge-invariant quantity $\Delta_{t}=\mu-\partial \psi_{0}$ falls off towards the limiting value $\Delta_{t}=1 / 2$ as $\sim t^{-1}$.

A definitive interpretation of how the discrete scale invariance arises in the model under investigation cannot be given just based on the numerical results presented, and it is not possible to determine what sets the corresponding scale. Starting from the observation (4.8), we propose an ansatz of the form

$$
\begin{aligned}
& \phi_{1}(t, z)=t^{v_{I, \phi}} \cos \left(v_{R} \log (t)\right) \tilde{\phi}(z)+\mathcal{O}\left(t^{x}, x<v_{I, \phi}<0\right), \\
& \phi_{2}(t, z)=t^{v_{I, \phi}} \sin \left(-v_{R} \log (t)\right) \tilde{\phi}(z)+\mathcal{O}\left(t^{x}, x<v_{I, \phi}<0\right), \\
& a_{t}(t, z)=\frac{Q}{z}+\mu+\mathcal{O}\left(t^{x}, x<0\right)
\end{aligned}
$$

and insert it into the equations of motion (2.6)-(2.9), using $-Q=\mu=\frac{1}{2}$ as throughout the paper. In this way, we obtain the lowest-order equation

$$
4 z^{2} h(z)\left(h^{\prime}(z) \tilde{\phi}^{\prime}(z)+h(z) \tilde{\phi}^{\prime \prime}(z)\right)+(z-1)^{2} \tilde{\phi}(z)=0
$$

for $\tilde{\phi}(z)$, which has the two independent solutions

$$
\tilde{\phi}_{\text {reg }}=\sqrt{\frac{2 z}{z+1}} P_{-\frac{1}{2}+\frac{i}{2}}\left(\frac{4 z}{z+1}-1\right) \text { and } \tilde{\phi}_{\text {irreg }}=\sqrt{\frac{z}{z+1}}{ }_{2} F_{1}\left(\frac{1}{2}-\frac{i}{2}, \frac{1}{2}+\frac{i}{2} ; 1 ; \frac{2 z}{z+1}\right),
$$

where $P_{n}(x)$ stands for the Legendre polynomial of the first kind and ${ }_{2} F_{1}$ is the hypergeometric function. We find that $\tilde{\phi}_{\text {irreg }}$ diverges at the event horizon $z=z_{H}=1$, and

\footnotetext{
${ }^{8}$ Note, however, that in many of these examples it is not the time variable $t$ in which log-periodic oscillations is observed.
} 
consequently the physical solution to (4.18) is given by const. $\times \tilde{\phi}_{\text {reg }}$, with no further parameter to adjust boundary conditions. The boundary expansion of $\tilde{\phi}_{\text {reg }}$ reads

$$
\tilde{\phi}_{\text {reg }}(z) \sim \text { const. } \times \sqrt{z}\left(\log (z)+\left(H_{-\frac{1}{2}+\frac{i}{2}}+H_{-\frac{1}{2}-\frac{i}{2}}+\log (2)\right) \ldots\right),
$$

implying

$$
\kappa_{1}=\frac{1}{H_{-\frac{1}{2}+\frac{i}{2}}+H_{-\frac{1}{2}-\frac{i}{2}}+\log (2)}=\kappa_{c},
$$

see equations (2.15) and (2.19). We have hence proven that a power law ansatz such as (4.16) can only solve the equations of motion if the boundary condition is fixed at the critical value $\kappa=\kappa_{c}$. However, the lowest-order equations obtained via the ansatz (4.16) do not fix the parameters $v_{I, \phi}$ and $v_{R, \phi}$. Presumably this would require the appropriate inclusion of higher orders into the ansatz.

The analytic study of power law tails of QNMs is an important subject, for example concerning the question whether these tails are intrinsically non-linear phenomena, see again [38] for an overview. There exist specialised analytical methods to treat these problems [50], and the methods employed in [44, 45] may also have applicability to our system. Due to the possible importance of higher-order effects, a full analytical analysis of the emerging log-periodic behaviour of our system is however beyond the scope of the present paper, and will be left to future investigations.

\section{Summary and outlook}

\subsection{Summary}

We have studied quantum quenches in a holographic Kondo model. We solved the full timedependent dynamics of our model numerically using spectral and finite difference methods, and we studied the relaxation of the system under various quench protocols. We found that the relaxation is determined by the lowest QNM of the initial state of the system, which describes an exponential decay, with an additional oscillatory profile for quenches to the normal phase. The lowest QNM provides an excellent description of the relaxation of the system not only at late times, as one expects close to equilibrium, but also almost immediately as the system begin to relax after the quench of the Kondo coupling: there does not appear to be any appreciable region in the onset of the relaxation that is not described by the lowest QNM. This seems to be a generic feature in holographic systems modelling strong dynamics. Most importantly, we found that in the condensed (screened) phase, the leading QNM is purely imaginary, which corresponds to over-damping. In a temperature region below $T_{c}$, we have $\omega \propto-i\langle\mathcal{O}\rangle^{2}$. This is consistent with expectations from the behaviour of the Kondo resonance [18]. At low temperatures, we see a deviation from mean-field behaviour, $\omega \propto-i \log (\langle\mathcal{O}\rangle)$.

The time-dependence of the flux through $A d S_{2}$ at the black hole horizon, which is dual to the size of the impurity representation, describes the decrease of degrees of freedom after a quench to the condensed phase. This corresponds to a measure for the formation of the 




(a) Log-linear plot of $\langle\mathcal{O}\rangle$ vs. time.



(c) Log-log plot of $|\langle\mathcal{O}\rangle|$ vs. time.



(b) Log-log plot of $\langle\mathcal{O}\rangle$ vs. time.

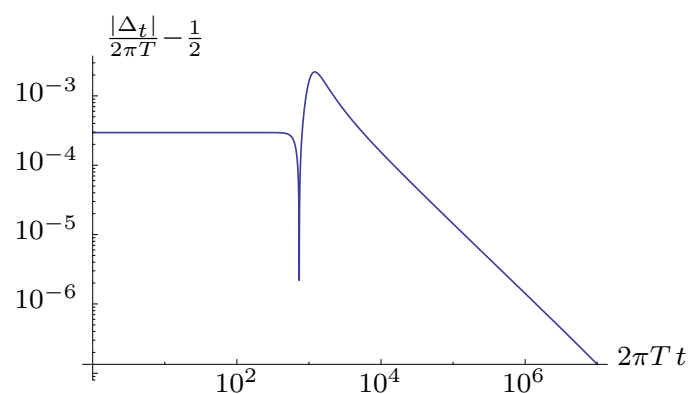

(d) Log-log plot of $\Delta_{t}$ vs. time.

Figure 11. A log-linear (a) and $\log -\log (\mathrm{b})$ plot of $\alpha_{1}$ and $\alpha_{2}$ as functions of time. Below, we see $\log$ - $\log$ plots of the gauge-invariant quantities $\Delta_{t}=\mu-\partial \psi_{0}$ and $\beta=\langle\mathcal{O}\rangle / N$ which are not featuring any oscillations in (logarithmic) time.

Kondo cloud at the site of the impurity. We found the decrease of degrees of freedom to be exponential.

In section 4.1 we studied in more detail the critical slowing down of the system near the phase transition at $T=T_{c}$, and showed the corresponding (combination of) critical exponents to be $z \nu=1$, just as expected from the similar holographic models [24, 30-33]. However, we also pointed out that due to the low dimensionality of our model, great care is required when interpreting critical exponents and their (hyper) scaling relations. Interestingly, the critical exponents of a large $N$ Kondo-Heisenberg lattice near a quantum critical point have been studied in [51, 52], with the result $z=3$.

Section 4.2 was then denoted to the study of quenches that lead directly to the critical point, $\kappa \rightarrow \kappa_{c}$. Our numerical results for quenches of this type, displayed in figure 11, show a damped log-periodic behaviour of the form (4.8). This log-periodic behaviour is known to be a telltale sign of discrete scale invariance, reviewed in [40] and observed in holographic models already in [46-48]. Unfortunately our numerics alone do not offer insight into the underlying mechanism causing the emergence of this phenomenon, and a lowest-order ansatz for the late time solutions of the equations of motion does not fix the involved complex critical exponent. A full analytical treatment of this interesting problem is hence left for future study. We note that in $[46,47]$, it was speculated that the emerging discrete 
scale invariance is connected to the physics of a near-horizon $A d S_{2}$ region. Similarly, in our model, we are effectively working with an asymptotic $A d S_{2}$ spacetime. This seems to imply that an $A d S_{2}$ structure is advantageous to the emergence of discrete scale invariance in holographic model. This may have interesting implications for the Sachdev-Ye-Kitaev (SYK) model [53-55]. We note that the SYK model and the model considered in the present paper are related by the fact that the Sachdev-Ye model of [53] is derived by writing a spin Hamiltonian in terms of the Abrikosov pseudo-fermions $\chi$ also used in the context of this paper, and reducing to an effective model in $0+1$ dimensions by appropriate averaging. This results in a quartic model for the $\chi$ 's. The major difference between the model of the present paper and the SYK model is that here, we break conformal symmetry explicitly by switching on the double-trace Kondo operator. This is a prerequisite for observing the phase transition described, which consequently is not present in the SYK model.

\subsection{Outlook}

One obvious generalisation of our approach will be to investigate quenches and other timedependent phenomena in the holographic two-impurity Kondo model of [16]. Moreover, it will be instructive to study the $T=0$ limit in further detail. This requires to add a quartic term to the scalar potential in order to ensure a well-defined IR fixed point and a finite condensate for $T \rightarrow 0$ (see also [14]).

Also, as pointed out above, the emergence of discrete scale invariance for critical quenches $\kappa \rightarrow \kappa_{c}$ deserves further study. In fact, we see from figure $8 \mathrm{~b}$ that in the condensed phase $\omega_{I} \rightarrow 0$ not only for $T / T_{c} \rightarrow 1$, but also for $T / T_{c} \rightarrow 0$. This seems to indicate that in the holographic Kondo model, critical slowing down does not only occur near the phase transition at $T=T_{c}$, but also at $T=0$. Whether this critical slowing down at zero temperature will be accompanied by similar log-periodic oscillations to those that we found at $T=T_{c}$ is however not clear. This requires a further study of the $T \rightarrow 0$ limit in a model which ensures stability of the IR fixed point, as described above.

One interesting further direction may be to combine the study of time-dependent phenomena in the holographic Kondo model of $[8,16]$ that was carried out in this work with the study of backreaction and entanglement entropy done in [13-15]. In particular, in $[14,15]$, it was shown that in the backreacted holographic Kondo model, there is a natural geometric length scale that takes the role of the Kondo scale $\xi_{K}$. A study of a holographic model allowing for both time-dependence and backreaction may hence allow the study of the characteristic time and length scales involved in the formation of the Kondo cloud in a similar way to what was done in [23] on the field theory side. Futhermore, calculations of the time-dependence of entanglement entropy after a quench in the holographic Kondo model may be compared to the results of field theory studies such as [22].

Finally, recently there appeared models of Kondo physics in the context of QCD [56] and Lorentz violation [57]. By adapting the methods of this paper, we expect that quenches in these models can also be studied holographically. 


\section{Acknowledgments}

We are very grateful to Paul Chesler and to Julian Sonner for sharing their insights about numerical analysis of time-dependent problems in holography. Furthermore we would like to thank Carlos Hoyos, Nina Miekley, Andy O'Bannon, Ioannis Papadimitriou and Jonas Probst for illuminating discussions. MF was supported by NCN grant 2012/06/A/ST2/00396.

\section{A Gauge fixing, coordinates and field redefinition}

We apply the radial gauge for both the gauge field $a$ on the $(1+1)$-dim. defect manifold as well as the Chern-Simons field in the $(2+1)$-dim. bulk. Regularity of the Chern-Simons field implies $\left.A_{t}\right|_{z_{H}}=0$ which prolongs to $A_{t}=0$ throughout the bulk after imposing the equations of motion. Hence only $A_{x}$ is non-trivial. As it is orthogonal to the defect manifold, it does not appear in the equations of motion for neither the scalar nor the gauge field. Thus we may neglect the Chern-Simons field in our analysis.

As already mentioned around eq. (2.14), the asymptotic behaviour of the scalar field in Schwarzschild-like coordinates is given by

$$
\phi_{i}(t, z) \approx \sqrt{z}\left(\alpha_{i}(t) \log (z)+\beta_{i}(t)\right)+O\left(z^{3 / 2}\right)
$$

as we approach the AdS boundary at $z=0$. The difficulty in dealing with the equations numerically is this non-analytic boundary behaviour.

We address this issue with a field redefinition and a change of coordinates. The change of variables can get rid of all (non-analytic at $z=0$ ) $\sqrt{z}$ terms in the boundary expansion if we let $y^{2}=z$. With this definition the boundary and horizon are at $y=0$ and $y=\sqrt{z_{H}}$, respectively. This redefinition only changes the log terms by a factor of two, however, so the fields are still non-analytic at $y=0$. In terms of the old coordinates, we define the new differentials to be

$$
\mathrm{d} t=\mathrm{d} v+\frac{2 y \mathrm{~d} y}{h(y)}, \quad \mathrm{d} z=2 y \mathrm{~d} y .
$$

The metric (2.1) becomes

$$
d s^{2}=\frac{1}{y^{4}}\left(-h(y) \mathrm{d} v^{2}-4 y \mathrm{~d} v \mathrm{~d} y+\mathrm{d} x^{2}\right)
$$

where $h(y)=1-y^{4}$. Note that $v$ and $t$ have the same level sets at the boundary, $y=0$, so that we can simply replace them when analysing boundary properties. The radial gauge $a_{z}=0$ in Schwarzschild-like coordinates translates into $a_{y}=2 y a_{v} / h(y)$ in our adapted EF-like coordinates. We decompose the scalar field into its real and imaginary part $\Phi=\phi_{1}+i \phi_{2}$. Applying this ansatz, the relevant equations of motion (2.6)-(2.9) are 
explicitly given by

$$
\begin{aligned}
0= & \partial_{y}^{2} \phi_{1}-\frac{4 \partial_{y} \partial_{v} \phi_{1}}{h(y)}-\frac{\left(1+3 y^{4}\right) \partial_{y} \phi_{1}}{y h(y)}-\frac{2 y\left(\phi_{2} \partial_{y} a_{v}+2 a_{v} \partial_{y} \phi_{2}\right)}{h(y)}, \\
0= & \partial_{y}^{2} \phi_{2}-\frac{4 \partial_{y} \partial_{v} \phi_{2}}{h(y)}-\frac{\left(1+3 y^{4}\right) \partial_{y} \phi_{2}}{y h(y)}+\frac{2 y\left(\phi_{1} \partial_{y} a_{v}+2 a_{v} \partial_{y} \phi_{1}\right)}{h(y)}, \\
0= & \partial_{y}^{2} a_{v}+\frac{3}{y} \partial_{y} a_{v}-\frac{4}{y^{3}}\left(\phi_{2} \partial_{y} \phi_{1}-\phi_{1} \partial_{y} \phi_{2}\right), \\
0= & \partial_{y} \partial_{v} a_{v}+\frac{4}{y^{3}} a_{v}\left(\phi_{1}^{2}+\phi_{2}^{2}\right) \\
& -\frac{2}{y^{4}}\left(\left[h(y) \partial_{y} \phi_{1}+2 y \partial_{v} \phi_{1}\right] \phi_{2}-\phi_{1}\left[h(y) \partial_{y} \phi_{2}+2 y \partial_{v} \phi_{2}\right]\right),
\end{aligned}
$$

where, as anticipated, the Chern-Simons field does not enter the equations of motion of the fields restricted to the defect.

The next step is a field redefinition where we subtract a number of dominant log terms in the boundary expansion. If we subtract enough terms, the redefined fields will have regular second derivatives at $y=0$. This also means that the non-analytic contributions from the $\log$ terms only appear at higher order. By applying (2.15), the $v$-dependent boundary expansion of the remaining fields looks like

$$
\begin{aligned}
\phi_{i}(v, y) \approx & \beta_{i}(v) y\left(1+2 \kappa_{i}(v) \log y\right) \\
& +y^{3}\left(a_{i}^{(4)}(v) \log ^{4} y+\cdots+a_{i}^{(1)}(v) \log y+a_{i}^{(0)}(v)\right)+\ldots, \\
a_{v}(v, y) \approx & -\frac{1}{2 y^{2}}+\mu(v)+c^{(3)}(v) \log ^{3} y+\cdots+c^{(1)}(v) \log y \\
& +y^{2}\left(d^{(4)}(v) \log ^{4} y+\cdots+d^{(1)}(v) \log y+d^{(0)}(v)\right)+\ldots,
\end{aligned}
$$

where $a_{i}^{(k)}(v), c^{(k)}(v)$ and $d^{(k)}(v)$ are functions of $\beta_{i}(v), \kappa_{i}(v), \mu(v)$ and their derivatives. We choose to subtract all terms containing a $\log y$ and divergent terms up to $\mathcal{O}\left(y^{3}\right)$. In other words, we define

$$
\begin{aligned}
& \tilde{\phi}_{i}(v, y)=\frac{1}{y}\left(\phi_{i}(v, y)-s^{\left(\phi_{i}\right)}\right), \\
& \tilde{a}_{v}(v, y)=a_{v}(v, y)-s^{\left(a_{v}\right)},
\end{aligned}
$$

where

$$
\begin{aligned}
s^{\left(\phi_{i}\right)}= & 2 y \beta_{i}(v) \kappa_{i}(v) \log y+y^{3}\left(a_{i}^{(4)}(v) \log ^{4} y+\cdots+a_{i}^{(1)}(v) \log y\right), \\
s^{\left(a_{v}\right)}= & -\frac{1}{2 y^{2}}+c^{(3)}(v) \log ^{3} y+\cdots+c^{(1)}(v) \log y \\
& +y^{2}\left(d^{(4)}(v) \log ^{4} y+\cdots+d^{(1)}(v) \log y\right)+\ldots
\end{aligned}
$$

These tilded fields have regular second $y$-derivatives on the domain $0 \leq y \leq 1$. Moreover, the boundary value of $\tilde{\phi}_{i}$ is $\beta_{i}$ and the boundary value of $\tilde{a}_{v}$ is $\mu$. With these redefinitions the equations of motion become too long to reproduce on the page here. However, they involve only fields and derivatives that are regular, which provides some numerical stability. 


\section{B Numerical evolution scheme}

Our goal is to find solutions in which we give $\kappa_{1}$ a $v$-dependent profile. We always start from a static solution, so the assumption is that $\kappa_{1}$ is constant for all $v<0$. At $v=0$, $\kappa_{1}(v)$ becomes a time-dependent function which smoothly connects to the initial constant value. This function could be a Gaussian or a hyperbolic tangent, for example. By solving the equations of motion, we find the resulting motion of the fields and, consequently, the coefficients $\beta_{1}(v), \beta_{2}(v)$ and $\mu(v)$ in (A.9) and (A.8). We use an implicit Crank-Nicholson integration method in $v$ and pseudospectral methods in $y$.

The first step is to find the static solution to the equations of motion. For that, we get an initial guess for the solutions by using the shooting method: we choose the initial value of $\kappa_{1}$, then adjust $\beta_{1}, \beta_{2}$ and $\mu$ until integration of the solutions from boundary to horizon yields solutions that are regular everywhere. After that, we discretise the equations of motion and solve the resulting non-linear matrix equations with the output of the shooting method as initial guess. More precisely, the equations are discretised on Chebyshev-Lobatto collocation points, starting at $y=\epsilon$ and ending at $y=1-\epsilon$. Differential operators are replaced by pseudospectral differentiation matrices. For $N$ collocation points, the fields are discretised to $\left(\tilde{\phi}_{1}\right)_{1}, \ldots,\left(\tilde{\phi}_{1}\right)_{N}$ and similarly for $\tilde{\phi}_{2}$ and $\tilde{a}_{v}$.

Since we know the boundary expansion for each of the fields, the first component of each can be replaced by that boundary expansion up to a given order, which will be made up of terms containing $\beta_{1}, \beta_{2}, \kappa_{1}$ and $\mu$, only. We apply a numerical algorithm to solve for the $3 N$ components $\left(\tilde{\phi}_{1}\right)_{2}, \ldots,\left(\tilde{\phi}_{1}\right)_{N},\left(\tilde{\phi}_{2}\right)_{2}, \ldots,\left(\tilde{\phi}_{2}\right)_{N},\left(\tilde{a}_{v}\right)_{2}, \ldots,\left(\tilde{a}_{v}\right)_{N}, \beta_{1}, \beta_{2}$ and $\mu$.

For our purposes, we found that it is sufficient to consider $N=50$ points, $\epsilon=10^{-3}$ and to cut the boundary expansion at next-to-leading order.

Starting with the static solution found in the previous section, we can use a timemarching method to evolve the solutions in $v$. We discretise the spacetime in the $v$ direction, using an evenly-spaced grid of step size $\Delta v$. The equations are stiff, so we use implicit methods. We use Crank-Nicholson, which has an error that is second order in the $\Delta v$. We start with the static solution at $v_{0}$ and earlier, so that the fields and coefficients at $v_{i}$ equal the fields and coefficients at $v_{0}$ for $i \leq 0$. At each step we then calculate the new fields and coefficients at $v_{i+1}$ from the values on the previous time slices.

So far this is general. The Crank-Nicholson method specifies precisely how we calculate values on $v$-slice $v_{i+1}$. We use the equations of motion and discretise them in the following way. For $f \in\left\{\tilde{\phi}_{1}(\cdot, y), \tilde{\phi}_{2}(\cdot, y), \tilde{a}_{v}(\cdot, y), \beta_{1}, \beta_{2}, \mu\right\}$ we make the replacements

$$
\begin{aligned}
f(v) & \rightarrow f\left(v_{i+1}\right) \\
\partial_{v} f(v) & \rightarrow 2 \frac{f\left(v_{i+1}\right)-f\left(v_{i}\right)}{\Delta v}-\partial_{v} f\left(v_{i}\right) \\
\partial_{v}^{2} f(v) & \rightarrow 2 \frac{2 \frac{f\left(v_{i+1}\right)-f\left(v_{i}\right)}{\Delta v}-\partial_{v} f\left(v_{i}\right)}{\Delta v}-\partial_{v}^{2} f\left(v_{i}\right)
\end{aligned}
$$

where for each $v$-derivative at slice $v_{i+1}$ we use the substitution rules to make a replacement until the equations contain no $v$-derivatives evaluated at $v_{i+1}$. Note that even though the 
original equations were first order in $v$, the equations for the tilded fields contain higher derivatives of the coefficients $\beta_{1}, \beta_{2}, \kappa$ and $\mu$.

The discretised equations of motion are used as follows. We know the values of the fields and their derivatives at $v$-slice $i$. Initially we use the static solution and set all the $v$-derivatives to zero. If we substitute in these numerical values, the discretised equations of motion are then algebraic in values of $f$ at $v_{i+1}$. We solve for these new values, store them, and also use equations (B.1) to find the $v$-derivatives at $v_{i+1}$.

Solving for the field values at $v_{i+1}$ is similar to the static case. We first discretise the equations in the $z$-direction as well, getting $3 N$ equations. We then replace $\left(\tilde{\phi}_{1}\left(v_{i+1}\right)_{1},\left(\tilde{\phi}_{2}\left(v_{i+1}\right)\right)_{1}\right.$, and $\left(\tilde{a}_{v}\left(v_{i+1}\right)\right)_{1}$ with their $(v$-dependent) boundary expansions and solve for the $3 N$ components $\left(\tilde{\phi}_{1}\left(v_{i+1}\right)\right)_{2}, \ldots,\left(\tilde{\phi}_{1}\left(v_{i+1}\right)\right)_{N},\left(\tilde{\phi}_{2}\left(v_{i+1}\right)\right)_{2}, \ldots,\left(\tilde{\phi}_{2}\left(v_{i+1}\right)\right)_{N}$, $\left(\tilde{a}_{v}\left(v_{i+1}\right)\right)_{2}, \ldots,\left(\tilde{a}_{v}\left(v_{i+1}\right)\right)_{N}, \beta_{1}\left(v_{i+1}\right), \beta_{2}\left(v_{i+1}\right)$ and $\mu\left(v_{i+1}\right)$. We adjust $\Delta v$ for each quench such that the shortest time scale appearing in the respective run is well resolved. All of the discussed algorithms were implemented and computed in MAтнEмAтісA.

Open Access. This article is distributed under the terms of the Creative Commons Attribution License (CC-BY 4.0), which permits any use, distribution and reproduction in any medium, provided the original author(s) and source are credited.

\section{References}

[1] J.M. Maldacena, The large- $N$ limit of superconformal field theories and supergravity, Int. J. Theor. Phys. 38 (1999) 1113 [hep-th/9711200] [INSPIRE].

[2] S.S. Gubser, I.R. Klebanov and A.M. Polyakov, Gauge theory correlators from noncritical string theory, Phys. Lett. B 428 (1998) 105 [hep-th/9802109] [INSPIRE].

[3] E. Witten, Anti-de Sitter space and holography, Adv. Theor. Math. Phys. 2 (1998) 253 [hep-th/9802150] [INSPIRE].

[4] V. Balasubramanian, A. Bernamonti, J. de Boer, N. Copland, B. Craps, E. Keski-Vakkuri et al., Holographic Thermalization, Phys. Rev. D 84 (2011) 026010 [arXiv:1103.2683] [INSPIRE].

[5] A. Buchel, L. Lehner and R.C. Myers, Thermal quenches in $N=2^{*}$ plasmas, JHEP 08 (2012) 049 [arXiv: 1206.6785] [INSPIRE].

[6] K. Murata, S. Kinoshita and N. Tanahashi, Non-equilibrium Condensation Process in a Holographic Superconductor, JHEP 07 (2010) 050 [arXiv: 1005.0633] [INSPIRE].

[7] M.J. Bhaseen, J.P. Gauntlett, B.D. Simons, J. Sonner and T. Wiseman, Holographic Superfluids and the Dynamics of Symmetry Breaking, Phys. Rev. Lett. 110 (2013) 015301 [arXiv: 1207.4194] [INSPIRE].

[8] J. Erdmenger, C. Hoyos, A. O'Bannon and J. Wu, A Holographic Model of the Kondo Effect, JHEP 12 (2013) 086 [arXiv:1310.3271] [INSPIRE].

[9] N. Read and D. M. Newns, On the solution of the Coqblin-Schreiffer hamiltonian by the large-n expansion technique, J. Phys. C 16 (1983) 3273.

[10] P. Coleman and N. Andrei, Diagonalisation of the generalised anderson model, J. Phys. C 19 (1986) 3211. 
[11] T. Senthil, S. Sachdev and M. Vojta, Fractionalized Fermi liquids, Phys. Rev. Lett. 90 (2003) 216403 [cond-mat/0209144] [INSPIRE].

[12] E. Witten, Multitrace operators, boundary conditions and AdS/CFT correspondence, hep-th/0112258 [INSPIRE].

[13] J. Erdmenger, M. Flory and M.-N. Newrzella, Bending branes for DCFT in two dimensions, JHEP 01 (2015) 058 [arXiv:1410.7811] [INSPIRE].

[14] J. Erdmenger, M. Flory, C. Hoyos, M.-N. Newrzella and J.M.S. Wu, Entanglement Entropy in a Holographic Kondo Model, Fortsch. Phys. 64 (2016) 109 [arXiv:1511.03666] [InSPIRE].

[15] J. Erdmenger, M. Flory, C. Hoyos, M.-N. Newrzella and J.M.S. Wu, Holographic impurities and Kondo effect, Fortsch. Phys. 64 (2016) 322 [arXiv:1511.09362] [INSPIRE].

[16] A. O'Bannon, I. Papadimitriou and J. Probst, A Holographic Two-Impurity Kondo Model, JHEP 01 (2016) 103 [arXiv: 1510.08123] [INSPIRE].

[17] J. Erdmenger, C. Hoyos, A. O'Bannon, I. Papadimitriou, J. Probst and J.M.S. Wu, Holographic Kondo and Fano Resonances, arXiv:1611.09368 [INSPIRE].

[18] J. Erdmenger, C. Hoyos, A. O'Bannon, I. Papadimitriou, J. Probst and J.M.S. Wu, Two-point Functions in a Holographic Kondo Model, arXiv:1612.02005 [INSPIRE].

[19] R. Vasseur and J.E. Moore, Edge physics of the quantum spin hall insulator from a quantum dot excited by optical absorption, Phys. Rev. Lett. 112 (2014) 146804 [arXiv:1312.3568].

[20] C. Kleine, J. Mußhoff and F.B. Anders, Real-time dynamics induced by quenches across the quantum critical points in gapless Fermi systems with a magnetic impurity, Phys. Rev. B 90 (2014) 235145 [arXiv: 1410.1770].

[21] M. Heyl and M. Vojta, Dynamics of symmetry breaking during quantum real-time evolution in a minimal model system, Phys. Rev. Lett. 113 (2014) 180601 [arXiv:1401.1743].

[22] D.M. Kennes, V. Meden and R. Vasseur, Universal quench dynamics of interacting quantum impurity systems, Phys. Rev. B 90 (2014) 115101 [arXiv:1406.5308] [INSPIRE].

[23] M. Nuss, M. Ganahl, E. Arrigoni, W. von der Linden and H.G. Evertz, Nonequilibrium, spatio-temporal formation of the Kondo screening-cloud on a lattice, Phys. Rev. B 91 (2015) 085127 [arXiv: 1409.0646].

[24] P. Basu, D. Das, S.R. Das and K. Sengupta, Quantum Quench and Double Trace Couplings, JHEP 12 (2013) 070 [arXiv: 1308.4061] [INSPIRE].

[25] N. Iqbal, H. Liu and M. Mezei, Quantum phase transitions in semilocal quantum liquids, Phys. Rev. D 91 (2015) 025024 [arXiv:1108.0425] [InSPIRE].

[26] H. Bantilan, F. Pretorius and S.S. Gubser, Simulation of Asymptotically AdS5 Spacetimes with a Generalized Harmonic Evolution Scheme, Phys. Rev. D 85 (2012) 084038 [arXiv: 1201.2132] [INSPIRE].

[27] T. Ishii, E. Kiritsis and C. Rosen, Thermalization in a Holographic Confining Gauge Theory, JHEP 08 (2015) 008 [arXiv: 1503.07766] [INSPIRE].

[28] I. Amado, M. Kaminski and K. Landsteiner, Hydrodynamics of Holographic Superconductors, JHEP 05 (2009) 021 [arXiv: 0903. 2209] [INSPIRE].

[29] P.C. Hohenberg and B.I. Halperin, Theory of Dynamic Critical Phenomena, Rev. Mod. Phys. 49 (1977) 435 [INSPIRE]. 
[30] K. Maeda, M. Natsuume and T. Okamura, Dynamic critical phenomena in the AdS/CFT duality, Phys. Rev. D 78 (2008) 106007 [arXiv:0809.4074] [INSPIRE].

[31] K. Maeda, M. Natsuume and T. Okamura, Universality class of holographic superconductors, Phys. Rev. D 79 (2009) 126004 [arXiv:0904.1914] [InSPIRE].

[32] M. Natsuume, Critical phenomena in the AdS/CFT duality, Prog. Theor. Phys. Suppl. 186 (2010) 491 [arXiv: 1006.4930] [INSPIRE].

[33] J. Sonner, A. del Campo and W.H. Zurek, Universal far-from-equilibrium Dynamics of a Holographic Superconductor, Nature Commun. 6 (2015) 7406 [arXiv:1406.2329] [INSPIRE].

[34] D.S. Fisher, M.P.A. Fisher and D.A. Huse, Thermal fluctuations, quenched disorder, phase transitions and transport in type-II superconductors, Phys. Rev. B 43 (1991) 130 [INSPIRE].

[35] N.D. Mermin and H. Wagner, Absence of ferromagnetism or antiferromagnetism in one-dimensional or two-dimensional isotropic Heisenberg models, Phys. Rev. Lett. 17 (1966) 1133 [INSPIRE].

[36] S.R. Coleman, There are no Goldstone bosons in two-dimensions, Commun. Math. Phys. 31 (1973) 259 [INSPIRE].

[37] P. Coleman, Introduction to Many-Body Physics, Cambridge University Press, Cambridge U.K. (2015).

[38] R.A. Konoplya and A. Zhidenko, Quasinormal modes of black holes: From astrophysics to string theory, Rev. Mod. Phys. 83 (2011) 793 [arXiv:1102.4014] [inSPIRE].

[39] G.T. Horowitz and V.E. Hubeny, Quasinormal modes of AdS black holes and the approach to thermal equilibrium, Phys. Rev. D 62 (2000) 024027 [hep-th/9909056] [INSPIRE].

[40] D. Sornette, Discrete scale invariance and complex dimensions, Phys. Rept. 297 (1998) 239 [cond-mat/9707012] [INSPIRE].

[41] G. Roósz, U. Divakaran, H. Rieger and F. Iglói, Nonequilibrium quantum relaxation across a localization-delocalization transition, Phys. Rev. B 90 (2014) 184202 [arXiv:1407.7829].

[42] M.W. Choptuik, Universality and scaling in gravitational collapse of a massless scalar field, Phys. Rev. Lett. 70 (1993) 9 [InSPIRE].

[43] A.M. Abrahams and C.R. Evans, Critical behavior and scaling in vacuum axisymmetric gravitational collapse, Phys. Rev. Lett. 70 (1993) 2980 [INSPIRE].

[44] E.W. Hirschmann and D.M. Eardley, Universal scaling and echoing in gravitational collapse of a complex scalar field, Phys. Rev. D 51 (1995) 4198 [gr-qc/9412066] [InSPIRE].

[45] E.W. Hirschmann and D.M. Eardley, Critical exponents and stability at the black hole threshold for a complex scalar field, Phys. Rev. D 52 (1995) 5850 [gr-qc/9506078] [INSPIRE].

[46] H. Liu, J. McGreevy and D. Vegh, Non-Fermi liquids from holography, Phys. Rev. D 83 (2011) 065029 [arXiv:0903.2477] [INSPIRE].

[47] T. Faulkner, H. Liu, J. McGreevy and D. Vegh, Emergent quantum criticality, Fermi surfaces and $A d S_{2}$, Phys. Rev. D 83 (2011) 125002 [arXiv:0907.2694] [INSPIRE].

[48] S.A. Hartnoll, D.M. Ramirez and J.E. Santos, Thermal conductivity at a disordered quantum critical point, JHEP 04 (2016) 022 [arXiv: 1508.04435] [INSPIRE].

[49] H. Georgi, Physics Fun with Discrete Scale Invariance, arXiv:1606.03405 [INSPIRE]. 
[50] E.S.C. Ching, P.T. Leung, W.M. Suen and K. Young, Wave propagation in gravitational systems: Late time behavior, Phys. Rev. D 52 (1995) 2118 [gr-qc/9507035] [INSPIRE].

[51] I. Paul, C. Pépin and M. R. Norman, Kondo breakdown and hybridization fluctuations in the kondo-heisenberg lattice, Phys. Rev. Lett. 98 (2007) 026402 [math/0605152].

[52] I. Paul, C. Pépin and M. R. Norman, Multiscale fluctuations near a kondo breakdown quantum critical point, Phys. Rev. B 78 (2008) 035109 [arXiv:0804.1808].

[53] S. Sachdev and J. Ye, Gapless spin fluid ground state in a random, quantum Heisenberg magnet, Phys. Rev. Lett. 70 (1993) 3339 [cond-mat/9212030] [INSPIRE].

[54] S. Sachdev, Holographic metals and the fractionalized Fermi liquid, Phys. Rev. Lett. 105 (2010) 151602 [arXiv: 1006.3794] [INSPIRE].

[55] J. Maldacena and D. Stanford, Comments on the Sachdev-Ye-Kitaev model, Phys. Rev. D 94 (2016) 106002 [arXiv:1604.07818] [INSPIRE].

[56] S. Ozaki, K. Itakura and Y. Kuramoto, Magnetically induced QCD Kondo effect, Phys. Rev. D 94 (2016) 074013 [arXiv: 1509.06966] [INSPIRE].

[57] D. Bazeia, F. A. Brito and J. C. Mota-Silva, Kondo effect from a Lorentz-violating domain wall description of superconductivity, Phys. Lett. B 762 (2016) 327 [arXiv:1606.07051] [INSPIRE]. 